\title{
1 Pyramidal tract neurons drive feed-forward excitation of striatum through
}

\section{2 cholinergic interneurons}

3 Nicolás A. Morgenstern $n^{1,}{ }^{*}$, Ana Filipa Isidro ${ }^{1}$, Inbal Israely ${ }^{2}$, and Rui M. Costa ${ }^{1,3,4,{ }^{*}}$

4

$5 \quad{ }^{1}$ Champalimaud Research, Champalimaud Centre for the Unknown, Lisbon 1400-038, Portugal

$6 \quad{ }^{2}$ Departments of Pathology \& Cell Biology, and Neuroscience, in the Taub Institute for Research on

$7 \quad$ Alzheimer's Disease and the Aging Brain, Columbia University, New York, NY 10027, USA.

$8 \quad{ }^{3}$ Departments of Neuroscience and Neurology, Zuckerman Mind Brain Behavior Institute, Columbia

$9 \quad$ University, New York, NY 10027, USA.

$10 \quad{ }^{4}$ Lead Contact

$11{ }^{*}$ Correspondence: nicolas.morgenstern@neuro.fchampalimaud.org (N.A.M.), rc3031@columbia.edu 12 (R.M.C.). 


\section{Highlights}

- PT, but not IT, corticostriatal inputs convey biphasic excitation to SPNs via a disynaptic circuit involving striatal Chls.

17

- PT neurons recruit Chls more efficiently than IT neurons due to a stronger relative input to Chls.

- The second phase of SPN excitation is mediated by nicotinic receptors at long-range glutamatergic axons in the striatum. result in delayed spiking. 


\section{Summary}

27

28

\section{Keywords}

41 Corticostriatal connectivity, spiny projection neurons, intratelencephalic, pyramidal tract, cholinergic

Corticostriatal connectivity is central for many cognitive and motor processes, such as reinforcement or action initiation and invigoration. The cortical input to the striatum arises from two main cortical populations: intratelencephalic (IT) and pyramidal tract (PT) neurons. We uncovered a new feedforward excitatory circuit, supported by a polysynaptic motif from PT neurons to cholinergic interneurons (Chls) to excitatory inputs, which runs in parallel to the canonical monosynaptic corticostriatal connection. This new motif conveys a delayed second phase of excitation to striatal spiny projection neurons (SPNs), through an acetylcholine-dependent glutamate release mechanism, resulting in biphasic corticostriatal signals. These biphasic signals are a hallmark of PT, but not IT, corticostriatal inputs, due to a stronger relative input from PT neurons to Chls. These results uncover a novel feed-forward circuit mechanism by which PT activity differentially gates excitatory inputs to the striatum, with potential implications for behavior, plasticity and learning. interneurons, circuit mapping, optogenetic, synapses, electrophysiology, striatum. 
43

\section{Introduction}

In the brain, the connection from the cortex to the striatum is central for many cognitive and motor processes, such as learning new motor skills or selecting proper actions in response to internal or contextual changes (Graybiel et al., 1994; Pennartz et al., 2009; Klaus et al., 2019). The striatum is the largest input nucleus to the basal ganglia, a group of interconnected subcortical nuclei that regulate brainstem, midbrain and thalamocortical circuits, forming a long-range connectivity loop with the later (Alexander and Crutcher, 1990). In the striatum, about $95 \%$ of the neurons are GABAreleasing spiny projection neurons (SPNs) (Kemp and Powell, 1971; Gerfen and Wilson, 1996). Besides being the most abundant ones, SPNs are the only neuronal subtype projecting outside this structure (Kemp and Powell, 1971), filtering the information that is outputted to downstream basal ganglia nuclei and, ultimately, modulating brainstem activity, and thalamic and cortical feed-back.

Synaptic input to striatum arises from most cortical areas, and to a lesser extent from the thalamus, through highly organized excitatory long-range axons (Yeterian and Van Hoesen, 1978; Flaherty and Graybiel, 1993; Wall et al., 2013; Guo et al., 2015; Hintiryan et al., 2016; Hooks et al., 2018; Johansson and Silberberg, 2020). Given that the striatum lacks intrinsic glutamatergic neurons (Tepper et al., 2007), these inputs onto SPNs and other striatal neuronal subtypes, are key for normal striatal function. Corticostriatal inputs are thought to convey motor and contextual signals to SPNs, information that is critical for proper action selection (Gremel and Costa, 2013; Klaus et al., 2019). Corticostriatal contacts are also the site for plasticity underlying striatal-dependent learning (Kreitzer and Malenka, 2008; Lee et al., 2015). In fact, synaptic weight changes occur in excitatory contacts onto SPNs when mice learn a motor task (Yin et al., 2009; Shan et al., 2014). Moreover, movement disorders in humans and mouse models of diseases like Parkinson's affect this connection (Stephens et al., 2005; Day et al., 2006; Fieblinger et al., 2014; Hawes et al., 2015), highlighting its importance. 
major categories: intratelencephalic (IT) and pyramidal tract (PT) neurons (Cowan and Wilson, 1994;

Molnár and Cheung, 2006; Shepherd, 2013). Each subpopulation has characteristic intracortical laminar position and stereotyped axonal projection patterns, suggesting divergent functionality (Beloozerova et al., 2003; Graybiel, 2005; Reiner, 2010; Li et al., 2015). The somata of IT neurons span cortical L5 and their axons extend within ipsi- and contralateral cortical areas, and also to the ipsi- and contralateral striatum. On the other hand, PT somata are located in deep L5 and their corticofugal projections send collaterals to several ipsilateral subcortical structures, predominating those to the ipsilateral striatum (Nelson et al., 2020). Moreover, while IT axons synapse onto PTs, direct PT contacts onto ITs are rare, suggesting a hierarchical IT $\rightarrow$ PT anatomo-functional organization (Morishima, 2006; Brown and Hestrin, 2009; Anderson et al., 2010; Kiritani et al., 2012). These morphological and connectivity features, together with studies showing that IT and PT have distinct roles in action planning and execution (Li et al., 2015), suggest that ITs are mostly involved in intracortical action preparation, while PTs trigger action execution by broadcasting a command signal throughout the multiple motor-related subcortical structures they innervate.

The striatum is therefore the only non-cortical structure where IT and PT pathways converge (Shepherd, 2013), synapsing onto both striatonigral and striatopallidal SPNs (Kress et al., 2013). Thus, understanding the differences between the signals that SPNs receive from these two key cortical afferents, and their impact on striatal output, becomes crucial for tackling neuronal circuits supporting motor learning and behavior.

However, the direct cortex $(C x) \rightarrow S P N$ connection is not the sole determinant of striatal output. In fact, SPN spiking is tightly controlled by intrastriatal polysynaptic interactions with sparse local GABAergic and cholinergic interneurons (Chls)(Assous and Tepper, 2018). For instance, parvalbumin-expressing fast-spiking interneurons (FSIs), driven by cortical inputs, exert strong feed-forward inhibition onto SPNs, controlling their output (Koós and Tepper, 1999; Mallet et al., 2005; Gittis et al., 2010; Planert et al., 2010). In turn, Chls regulate striatal function by releasing acetylcholine that acts either through 
neuromodulatory muscarinic receptors onto SPNs (Goldberg et al., 2012) or through presynaptic nicotinic receptors regulating dopamine, GABA and glutamate release (Wonnacott et al., 2000; influence on SPNs spiking, it is still unknown whether striatal interneurons have biased inputs from IT versus PT neurons.

In this study, we used transgenic mouse lines, optogenetics and slice electrophysiology to investigate the differences between IT and PT corticostriatal connectivity to the striatum. We found a new feedforward excitatory circuit, supported by a polysynaptic motif from PT neurons to Chls to excitatory inputs, running in parallel to the canonical monosynaptic $C_{x} \rightarrow S P N s$ connection. This new motif conveys a delayed second phase of excitation to SPNs, through an acetylcholine-dependent glutamate release mechanism, resulting in biphasic corticostriatal signals. Moreover, we found that these biphasic signals are a hallmark of PT, but not IT, corticostriatal inputs, due to their stronger relative input to Chls. This work uncovers a novel circuit mechanism by which PT, but not IT neurons, drive feed-forward excitation to the striatum, with potential implications for behavior, plasticity and learning.

\section{PT corticostriatal inputs evoke biphasic responses onto SPNs.}

To investigate the differences between the signals that IT and PT corticostriatal neurons convey to SPNs, we crossed a transgenic mouse line expressing ChannelRhodopsin-2 (ChR2)-EYFP under the control of cre-recombianse (Ai32; Rosa-CAG-LSL-ChR2(H134R)-EYFP-WPRE) with a mouse line 
expressing cre-recombinase in either IT (TIx3; Tg(T/x3-cre)PL58Gsat/Mmucd) or PT (OE25; Tg(Chrna2cre)OE25Gsat/Mmucd) cortical neurons. This resulted in the selective expression of ChR2-EYFP in one of these two neuronal populations (IT-ChR2-EYFP and PT-ChR2-EYFP, respectively), confirming their laminar location in cortical L5, as well as their long-range axonal projections into the dorsolateral striatum (DLS, Figure $1 A$ and B). We then used whole-cell patch-clamp to record excitatory postsynaptic currents (EPSCS) from SPNs in the DLS of acute brain coronal slices while wide-field photostimulating pathway-specific corticostriatal fibers (Figure 1C). This is a well-established approach to study long-range connectivity in vitro, because ChR2-expressing axons remain photoexcitable despite losing branches or the connection to their parental soma during the slicing process (Petreanu et al., 2007, 2009; Yang et al., 2013; D'Souza et al., 2016; Morgenstern et al., 2016;

Tanimura et al., 2019). As expected, presynaptic photostimulation of identical power but different durations elicited postsynaptic responses of variable amplitude (Figure 1D). Surprisingly, IT and PT activation elicited responses with different characteristics. The stimulation of IT fibers evoked typical monophasic EPSCs, consistent with direct excitatory inputs from IT neurons. In turn, PT axons activation often elicited EPSCs with two distinguishable phases, suggesting an additional disynaptic component (Figure 1D). Accordingly, when comparing IT $\rightarrow$ SPN with PT $\rightarrow$ SPN EPSCs from individual trials showing first peaks of similar amplitude, the overall charge transferred to postsynaptic SPNs was larger for PT $\rightarrow$ SPNs (Figure 1E). To investigate if such charge difference was due to the higher probability of evoking a second peak in PT $\rightarrow$ SPN EPSCs, we used a threshold to detect the delayed phase of corticostriatal signals (Methods). Indeed, the probability of evoking a second phase on SPN EPSCs was higher for PT than for IT stimulation across all first peak amplitudes explored (Figure 1F). Moreover, both the amplitude and the charge of the second phase increased with the increase in amplitude of the first peak (Figures $\mathbf{1 G}$ and $\mathbf{H}$ ). We standardized comparisons to the amplitude of the EPSC first peak rather than to the phostostimulation conditions because of the variable expression levels of ChR2 across slices, subjects and transgenic mouse lines. However, although PT fibers required, on average, longer illumination times than IT axons for evoking responses within the 
explored range (IT, $6.05 \pm 0.28 \mathrm{~ms}$, range: $0.1-30, \mathrm{n}=423 ; \mathrm{PT}, 9.24 \pm 0.17 \mathrm{~ms}$, range: $0.3-22, \mathrm{n}=797$; $\mathrm{p}=1.01 \times 10^{-37}$, Wilcoxon rank sum test, $\left.\mathrm{z}=-12.84\right)$, when comparing similar photostimulation conditions, the probability of evoking biphasic EPSCs was higher for PT inputs (Figure 11).

To better characterize the differences between IT and PT corticostriatal signals, we then measured the latencies from the start of illumination to the first and second peak of the EPSCs (Figures $\mathbf{1 J}$ and K). We found that the overall latency to the first peak was shorter for PT $\rightarrow$ SPNs when compared to IT $\rightarrow$ SPNs (PT: $14.91 \pm 0.14 \mathrm{~ms}, \mathrm{n}=797$ trials; IT: $18.44 \pm 0.24 \mathrm{~ms}, \mathrm{n}=423$ trials; $\mathrm{p}=3.99 \times 10^{-60}$, Wilcoxon rank sum test, $\mathrm{z}=16.36)$. Moreover, this analysis showed a mean delay of $20.44 \pm 0.19 \mathrm{~ms}(\mathrm{n}=610$ tirals) from the first to the second peak evoked by PT photostimulation (Figure 1K, inset).

We next investigated how the findings from this dataset, displayed above in a trial-by-trial basis, were reflected at the level of individual neurons. Consistently, we found that, when comparing EPSCs in a similar range of first peak amplitudes, SPNs receive more charge from PT than from IT presynaptic neurons (Figure 1L). For this analysis, we first averaged five consecutives photostimulation trials, resulting in a mean EPSC for each individual SPN (Figure 1M). Furthermore, this approach allowed us to calculate the neuron-based EPSC second peak response probability. In line with the results above, we found a very low probability for evoking biphasic responses when activating IT fibers, but a highly reliable occurrence of EPSC second phases when stimulating PT inputs (Figure 1N).

We then designed an experiment to rule out the possibility that the EPSC second phase is elicited by ChR2-expressing long-range axons not originated in the cortex, resulting from extracortical expression of cre-recombinase in the PT-cre OE25 mouse line (www.gensat.org). For this purpose, we injected a cre-dependent AAV in the motor cortex of an OE25 mouse, restricting the expression of ChR2 to PT neurons in this area. Five weeks later, we recorded from a SPN in the DLS (Figure 10) and found that 1P). 
In summary, we found that IT and PT corticostriatal signals are different, with IT inputs evoking mostly monophasic responses, and PT inputs reliably eliciting two sequential excitatory signals that result in biphasic EPSCs onto SPNs.

Corticostriatal PT $\rightarrow$ SPN EPSC second peak is mediated by striatal Chls.

In light of our findings, we hypothesized that whereas the first IT and PT EPSC peak is mediated by direct cortical input to SPNs, the second phase reflects intrastriatal polysynaptic feed-forward excitation, preferentially recruited by PT long-range axons. We dismissed a potential contribution of intracortical polysynaptic interactions, because most cortical axons innervating the DLS are severed from their somas upon coronal slice preparation, due to the anatomy of cortical projections. In addition, focal presynaptic phostostimulation with a laser beam in the near vicinity of the recorded SPNs reliably evoked biphasic PT $\rightarrow$ SPN EPSCs (Supplemental Figure 1A), further supporting a role for local intrastriatal interactions in the late phase of the EPSC.

More than 95\% of striatal neurons are GABAergic SPNs (Kemp and Powell, 1971; Gerfen and Wilson, 1996). Moreover, with exception of a small population of acetylcholine-releasing Chls, all other local interneurons also release GABA (Assous and Tepper, 2018). Since in our experimental conditions SPNs were clamped at a membrane potential below the chloride reversal potential (SPN $V_{\text {holding }}=-80 \mathrm{mV}, E_{\mathrm{cl}}$ $=-75.6 \mathrm{mV}$ ), GABA receptor activation would result in excitatory rather than inhibitory currents (Plenz, 2003). For this reason, we first investigated whether GABAergic neurotransmission is underlying the second phase of PT $\rightarrow$ SPN excitation. For this purpose, we photostimulated PT axons while monitoring SPN EPSCS in the absence or presence of the $\mathrm{GABA}_{\mathrm{A}}$ receptor antagonist picrotoxin (PTX). Since the charge and amplitude of the EPSC second phase increase with the amplitude of the first peak (Figure $1 \mathbf{G}$ and $\mathbf{H}$ ), we calculated the charge ratio and the peak ratio to relate the magnitude of both phases (Methods). These normalized metrics, as well as the second peak response probability, resulted robust 
comparisons across trials within the same SPN, as well as across different SPNs. Strikingly, the addition of PTX to the extracellular solution had no effect on the charge ratio (Figures 2A, B and C). Even though PTX elicited a moderate decrease in the peak ratio (Figure 2D) the magnitude of such decrease was not enough to fully suppress the second phase of EPSCs in any of the recorded SPNs (Figures 2B and membrane was clamped above the chloride reversal potential (Supplemental Figure 1B), further supporting that it is not mediated by GABA.

Having ruled out the role of GABAergic interneurons, we postulated that Chls could mediate these local polysynaptic responses triggered by PT. In fact, current-voltage curves of PT $\rightarrow$ SPN second phase resembled cationic currents compatible with glutamate or acetylcholine acting through nicotinic receptors (Supplemental Figure 1 C and D). To test this, we recorded SPNs while photostimulating PT axons without or with the nicotinic acetylcholine receptor antagonist methyllycaconitine (MLA) in the bath. MLA significantly reduced the charge ratio, the peak ratio and the second peak response probability of PT $\rightarrow$ SPN responses (Figures $\mathbf{2 E - H}$ ), which partially recovered after MLA wash out on the first, phase of these EPSCs (Supplemental Figures $\mathbf{2}$ and 3), indicating that it did not reduce overall excitability. Moreover, when comparing the magnitude of the reduction exerted by MLA or PTX, we found a significantly stronger effect of the cholinergic blocker on both the charge ratio and the peak ratio (Figures $\mathbf{2}$ and $\mathrm{J}$ ). Furthermore, MLA reduced the PT $\rightarrow$ SPN EPSC second phase to undetectable levels in most of the recorded neurons (Figure $\mathbf{2 L}$ ), implicating Chls in these delayed signals. In summary, our data strongly suggests that the second excitatory component of the biphasic PT $\rightarrow$ SPN responses is mediated by nicotinic receptors that are activated upon the selective recruitment of local striatal Chls by PT input stimulation. 
In the context of our results, we wondered if different connectivity rules operating for each corticostriatal pathway could explain the preferential recruitment of Chl $\rightarrow$ SPN excitation by PT, but not by IT activation. In that sense, we hypothesized that the relative input strength to different striatal postsynaptic neuronal subtypes is differentially balanced for the IT and PT pathways. Thus, we designed an experiment to test whether cortical IT and PT neurons contact striatal Chls monosynaptically, and if so, how the connection strength from each pathway is distributed between Chls and SPNs.

In brains slices of IT- or PT-ChR2-EYFP mice, we first patched a Chl in the DLS (Figure 3A and B). We initially recorded visually identified putative Chls and confirmed their cholinergic identity by assessing their typical electrophysiological properties (depolarized resting membrane potential, presence of sag upon hyperpolarization, spike half-width $>1 \mathrm{~ms}$ and regular spiking; Figure $3 \mathrm{C}$ and Supplemental

Figure 4) in drug-free extracellular solution. We further confirmed the cholinergic phenotype of the recorded neurons by their characteristic larger somatic area (Chls: $239.86 \pm 11.79 \mu \mathrm{m}^{2}$, range: 146.32 - 356.6, $n=19 ;$ SPNs: $108.46 \pm 6.53 \mu \mathrm{m}^{2}$, range: $59.57-160.62, \mathrm{n}=20 ; \mathrm{p}=1.6 \times 10^{-7}$, Wilcoxon rank sum test, $z=-5.24$, Figure 3B) and their reactivity to Choline-Acetyl Transferase (ChAT) immunolabelling (16 out of 19 Chls were recovered and identified as $\mathrm{ChAT}^{+}$, Figure $\mathbf{3 C}$ ).

After that, we added 4-aminopiridine (4-AP) and tetradotoxine (TTX) to the extracellular solution. This drug combination suppresses spiking while preserving $C h R 2^{+}$synaptic terminals ability to release neurotransmitter when photostimulated, thus, restricting EPSCs only to monosynaptic contacts

(Petreanu et al., 2009; Yang et al., 2013; D’Souza et al., 2016; Morgenstern et al., 2016). In these conditions, we photostimulated IT or PT corticostriatal presynapses with a blue laser following a grid pattern over the dendrites of the postsynaptic ChI (Figure 3D). For each neuron, we computed the total input charge by summing all EPSCs. Since both IT and PT corticostriatal pathways stimulation 
comparable IT $\rightarrow$ Chl and PT $\rightarrow$ Chl EPSCs across experiments (IT: $6.77 \pm 1.01$ pC*10 ${ }^{3}$, range: $1.69-10.57$, $n=10 ;$ PT: $4.76 \pm 1.34 p C^{*} 10^{3}$, range: $1.31-11.32, n=9 ; p=0.2775$, Wilcoxon rank sum test). Ideally, we would have recorded first from SPNs and calibrated photostimulation to evoke similar IT $\rightarrow$ SPN and PT $\rightarrow$ SPN EPSCs. However, the need for confirming Chls electrophysiogical phenotype in the absence of drugs, forced us to record first from Chls. Right after assessing inputs to the Chl, we recorded a neighboring SPN (less than $100 \mu \mathrm{m}$ apart, Figure 3A, B and Supplemental Figure 4E) and repeated the photostimulation protocol with identical illumination conditions (Figure 3D). Since neighboring neurons could potentially sample from the same population of afferent axons, with this experimental design we could compare the pathway-specific input strength balance to ChI-SPN pairs, irrespective of ChR2 expression level variations across slices (Petreanu et al., 2009; Yang et al., 2013; D'Souza et al., 2016; Morgenstern et al., 2016).

We found that the input strength is differentially weighted for each corticostriatal pathway. While PT inputs exhibited similar strengths across Chls and SPNs (Figure 3E and F), the IT connection was significantly biased towards the SPNs (Figure $\mathbf{3 G}$ and $\mathbf{H}$ ). To further quantify how the connection strength of each pathway distributes between ChIs and SPNs, we normalized the corticostriatal input that a given ChI-SPN pair receive to the input of that SPN (Figure $3 F$ and $\mathbf{H}$ ). We found that the PT $\rightarrow$ ChI relative input strength is variable, with a population median of 1.66 times the PT $\rightarrow$ SPN input (Figure 3F). Contrarily, IT $\rightarrow$ Chl relative input strength was consistently weaker, with a population median of 0.28 times the IT $\rightarrow$ SPN in put (Figure $\mathbf{3 H}$ ). These data strongly suggest that IT and PT pathways follow different connectivity rules when synapsing onto striatal neurons. Moreover, the normalized PT $\rightarrow \mathrm{Chl}$ input was one order of magnitude stronger than the normalized IT $\rightarrow$ Chl input (Figure 3I), supporting a model where, provided similar input to SPNs, PT neurons are more likely to recruit Chls than IT neurons. Importantly, these differences in IT and PT connectivity could not be explained by differences in the size of the dendrites of the recorded Chls (Supplemental Figure 4G) nor by the distance between Chls and SPNs within the same pair (Supplemental Figure 4E). 
Our findings support the co-existence of two parallel excitatory corticostriatal circuits. On one hand, there is the canonical direct long-range connection from cortical neurons to SPNs, which photoactivation elicits the monosynaptic first phase of the IT $\rightarrow$ SPN and PT $\rightarrow$ SPN EPSCs. On the other hand, we described a novel parallel direct long-range projection to striatal Chls from both, IT and PT neurons, but with higher relative input strength for PT $\rightarrow$ Chls than for IT $\rightarrow$ Chls. As a consequence, only PT inputs effectively recruit Chls upon photostimulations that evoke similar EPSC first peak amplitudes onto SPNs. Together with our previous data, these results support a corticostriatal $\mathrm{Cx}_{\mathrm{X}} \rightarrow$ Chls $\rightarrow$ SPNs feed-forward excitatory motif, and explain why PT inputs are more likely than IT inputs to evoke biphasic corticostriatal EPSCs onto SPNs.

Chls indirectly excite SPNs via an acetylcholine-dependent glutamate release mechanism.

We next wanted to dissect the precise circuit mechanism underlying ChI $\rightarrow$ SPN EPSCs. One possibility is that Chls form direct monosynaptic contacts onto SPNs supporting nicotinic transmission, which existence, to our understanding, is not described in the literature (Zhou et al., 2002; Assous and Tepper, 2018). Alternatively, Chls could indirectly convey signals to SPNs by nicotinic activation of presynaptic glutamatergic axons (Campos et al., 2010; Howe et al., 2016; Abudukeyoumu et al., 2018). Interestingly, this interaction between cholinergic and glutamatergic systems is mediated by $\alpha 7$-nicotinc receptors, which are a major target of MLA, the cholinergic antagonist proved to suppress the second phase of PT $\rightarrow$ SPN EPSCs (Figure 2).

To investigate these two possibilities, we used a double transgenic mouse line (ChAT-Cre x Ai32) where

ChR2 is expressed in acetylcholine-releasing neurons (ChAT-ChR2-EYFP). Since the cholinergic innervation of the striatum is dominated by local Chls, rather than extrinsic innervation (English et al., 2012; Goldberg et al., 2012; Dautan et al., 2014; Deffains and Bergman, 2015), we reasoned that photostimulation in this preparation would mostly reflect the activation of striatal Chls (Figure 4A). 
We could reliably evoke EPSCs on the recorded SPNs when photoactivating Chls (Figure 4C and D).

Strikingly, when compared to the PT $\rightarrow$ SPN latency to the first peak, the ChI $\rightarrow$ SPN EPSC peak latency was longer (PT first peak: $14.91+0.14 \mathrm{~ms}, \mathrm{n}=797$; $\mathrm{Chl} \rightarrow$ SPN peak: $32.33+0.37 \mathrm{~ms}, \mathrm{n}=379$; Kruskalfrom PT $\rightarrow$ SPN second phase (Chl $\rightarrow$ SPN peak: $32.33+0.37 \mathrm{~ms}, \mathrm{n}=379$; PT second peak: $35.4+0.24 \mathrm{~ms}$, $n=610$, Kruskal-Wallis test and multicompare post hoc Dunn's test test, $p<0.0001$; Figure 4B), suggesting a delayed mechanism, more compatible with indirect transmission.

To confirm that these EPSCs were mediated by a similar circuit to PT $\rightarrow$ SPN EPSC second phase, and not by direct glutamate release from Chls (Higley et al., 2011), we tested the susceptibility of these responses to MLA. Both the charge and the amplitude of ChI $\rightarrow$ SPN EPSCs were significantly reduced by MLA (Figure 4C, E and F), indicating the necessity of nicotinic activation to excite SPNs.

We then reasoned that if ChI $\rightarrow$ SPN EPSCS are mediated by monosynaptic contacts; they should be the recruitment of presynaptic glutamatergic terminals, EPSCs should then be modulated by blocking glutamate receptors. Indeed, the addition of the AMPA and NMDA receptor blockers 6,7dinitroquinoxaline-2,3-dione (DNQX) and (2R)-amino-5-phosphonovaleric acid (APV), respectively, acetylcholine-dependent glutamate release from excitatory long-range axons innervating DLS.

\section{Activation of PT corticostriatal inputs evoke delayed SPN spiking.}

The data presented so far supports a new excitatory long-range corticostriatal circuit consisting of PT $\rightarrow$ Chls $\rightarrow$ glutamatergic axons (glutAx) $\rightarrow$ SPNs. We next investigated how this feed-forward excitation impacts on SPN spiking, and in turn, on striatal output. We started by photostimulating IT 
or PT fibers while monitoring the membrane potential of SPNs using current-clamp recordings. Not surprisingly, subthreshold responses from these experiments paralleled our previous findings using voltage-clamp (Figure 1). We found that excitatory postsynaptic potentials (EPSPs) of similar first peak amplitude had larger area when elicited by PT activation, both at the trial-by-trial (Figure 5A) and at the individual SPN level (Figure 5B). Consistently, we found that such area difference is due to biphasic EPSPs (Figure 5C), which occur with higher probability when PT, rather than IT, axons are activated (Figure 5D).

Although in the intact brain SPN spiking would probably depend on simultaneous IT and PT EPSCs, our approach gave us the unique opportunity to independently explore how IT or PT inputs control SPNs spiking, which is key to understanding corticostriatal connectivity in further detail. However, in normal ACSF conditions, only IT activation successfully evoked spiking at SPNs (Figure 5E). To overcame this, we reproduced the latter experiments in low-magnesium and high-calcium ACSF $\left(0 \mathrm{mM} \mathrm{Mg}^{2+}, 4 \mathrm{mM}\right.$ $\mathrm{Ca}^{2+}$ ) pursuing the facilitation of polysynaptic activity. In this preparation, IT and PT photostimulation evoked spiking in SPNs with similar probability (Figure 5E) but different latency. Overall latency from the start of the illumination until the spike peak revealed significantly longer for PT activation (IT, 20.57 $\pm 0.58 \mathrm{~ms}$, range: $15.9-30.8, \mathrm{n}=38 ; \mathrm{PT}, 34.48 \pm 1.16 \mathrm{~ms}$, range: $18.2-55.1, \mathrm{n}=52 ; \mathrm{p}=3.59 \times 10^{-12}$, Wilcoxon rank sum test, $z=-6.95)$. Interestingly, while IT $\rightarrow$ SPN spike latency resembled the timing to the first EPSC peak, the latency to PT $\rightarrow$ SPN spikes was similar to the second EPSC peak (Figure $1 \mathrm{~J}$ and K). However, spike latency differences could also emerge from differences in ChR2 expression levels in IT and PT lines. For instance, lower ChR2 expression levels in PT than in IT axons would delay the start of the PT $\rightarrow$ SPN EPSPs, shifting the peak of the action potentials towards the right. To rule out this possibility, we separately analyzed the latency from the beginning of the illumination until the EPSP start and from this point until the action potential peak (Methods). We found that while the latency until the beginning of the EPSP was indistinguishable between IT and PT (Figure 5F), PTtriggered spikes developed significantly later after that point (Figure 5G). These results indicate that, at least in these conditions, IT and PT presynaptic activation speed is comparable, and rule out the 
possibility that overall spiking latency differences are due to variable levels of ChR2 expression. Importantly, the overall light durations used for evoking IT- and PT-dependent spiking were similar (IT, $5.95 \pm 0.31 \mathrm{~ms}$, range: $3.5-10, n=38 ; \mathrm{PT}, 6.13 \pm 0.30 \mathrm{~ms}$, range: $3.5-10, n=52 ; p=0.8523$, Wilcoxon rank sum test, $z=-0.19)$, and when comparing identical light conditions, the latency from EPSP start until action potential peak was always longer for PT $\rightarrow$ SPN spikes than for IT $\rightarrow$ SPN spikes (Figure $\mathbf{5 H}$ ).

Altogether, this data indicates that while IT-triggered spiking occurs by the faster monosynaptic depolarization of SPNs, PT $\rightarrow$ SPNs direct depolarization is less likely to evoke suprathreshold responses, and spiking threshold is most likely reached during the delayed feed-forward Chls $\rightarrow$ glutAx $\rightarrow$ SPNs excitation phase. This becomes especially evident in conditions that favor polysynaptic activity, and it is reflected in the fact that PT $\rightarrow$ SPN spikes, in contrast to IT $\rightarrow$ SPN spikes, occur at the start of the EPSP second phase (Figure $5 \mathrm{I}$ and $\mathrm{J}$ ).

\section{Discussion}

Our data uncovers a new circuit mechanism by which IT and PT corticostriatal inputs differentially impact SPNs (Figure 5K). We found a feed-forward corticostriatal excitatory circuit, predominantly supported by the PT $\rightarrow$ Chls $\rightarrow$ glutAx $\rightarrow$ SPNs motif, running in parallel to the canonical excitatory monosynaptic connection from the cortex to SPNs. The photoactivation of that motif evokes a second phase of excitation onto SPNs, mediated by acetylcholine-induced activation of nicotinic receptors at presynaptic glutamatergic terminals in the DLS, and resulting in biphasic corticostriatal signals (Figure 
presented here provide new insights on the polysynaptic impact of corticostriatal IT and PT signals onto SPNs, with potential implications for movement, plasticity and learning.

Johansson and Silberberg, 2020). However, this connection was usually assumed homogeneous, neglecting the differences between the diversity of cortical inputs impinging onto striatal SPNs and interneurons. In that sense, previous functional studies using electrical or optogenetic stimulation of corticostriatal fibers and testing its impact onto SPNs, would have predominantly recruited the denser bilateral IT pathway, occluding the details of the sparser PT $\rightarrow$ SPN connection described here. We overcame that limitation by using mouse lines allowing population-specific control of axonal spiking (Gerfen et al., 2013). We found that, in parallel to the direct IT/PT $\rightarrow$ SPN connection (Kress et al., 2013), both IT and PT pathways contact Chls with different relative strength, indicating target selectivity of corticostriatal pathways. Interestingly, a recent study testing ipsi- and contralateral striatal innervation from motor and somatosensory cortices showed that Chls are exclusively contacted by ipsilateral axons, most likely reflecting predominant PT inputs. In that same study, FSIs, the canonical striatal feed-forward inhibitory interneurons (Mallet et al., 2005; Gittis et al., 2010) were proven highly innervated by bilateral corticostriatal fibers, indicating strong IT connectivity (Johansson and Silberberg, 2020). Such observation is suggestive of, at least, some degree of corticostriatal IT $\rightarrow$ FSIs and PT $\rightarrow$ Chls specificity, which is partially demonstrated here by our result showing stronger PT relative input strength to Chls (Figure 3I). Although further studies are necessary, this idea becomes especially relevant in the context of a model where ITs are preparatory and PTs broadcast the action command to many motor-related structures (Li et al., 2015). It is, then, tempting to speculate that ITs may permit action preparation by triggering up-states onto action-specific SPNs while silencing action-unrelated SPNs through FSIs. Subsequent PT signals might be key for transitioning from up-states to spikes in the action-related SPNs commanding execution. In this scenario, the feed-forward excitation exerted by PT $\rightarrow$ Chls $\rightarrow$ glutAx $\rightarrow$ SPNs, by boosting postsynaptic 
membrane depolarization and input integration, could help securing SPNs spiking and downstream information flow. A possible reason why this second excitatory phase was not detected in the scarce functional studies that have tested PT $\rightarrow$ SPN connectivity to date, may be on cortical neurons heterogeneity. Recent studies showed that both IT and PT populations could be genetically subdivided into several sub-classes with different synaptic targets (Economo et al., 2018; Tasic et al., 2018). Thus, since in those cases focal subcortical injections of retrogradely labeling virus were used to achieve PT expression of ChR2 (Kress et al., 2013), it is likely that in previous studies, only a subset of the neurons labelled in the PT-ChR2-EYFP mice were recruited by photostimulation. A similar scenario may underly the variable shape of the EPSCs we evoked when driving ChR2 expression with a viral injection in the cortex (Figure 1P). In any case, more research will be required to dissect which PT sub-classes are indeed preferentially targeting Chls. Understanding the intrastriatal connectivity of PT sub-classes would, for instance, reveal whether during the second EPSC phase, acetylcholine enhances glutamate release from the same axons driving Chls, or it acts onto other long-range afferents reaching DLS. In fact, exploring the selectivity of acetylcholine for gating different populations of presynaptic longrange axons innervating the DLS, will help elucidating whether PT, IT, thalamic or other presynaptic inputs, alone or in specific combinations, underlie the second phase of excitation reported here. computations.

Besides driving feed-forward excitation, the activation of the PT $\rightarrow$ Chls $\rightarrow$ glutAx $\rightarrow$ SPNs motif could gate a window for inducing long-lasting synaptic changes specifically at the activated $\mathrm{C}_{x} \rightarrow \mathrm{SPN}$ contacts, by increasing local levels of acetylcholine. Changes in acetylcholine concentrations are believed to determine the occurrence and the sign (potentiation or depression) of long-term plasticity upon some pre- and postsynaptic activation combinations through muscarinic receptors (Centonze et al., 1999; Calabresi et al., 2000; Shen et al., 2005) and by modulating dopamine levels (Wang et al., 2006; Shen et al., 2008; Threlfell et al., 2012). Dopamine has long been implicated as a key 
Yagishita et al., 2014) and acetylcholine plays a crucial role in the striatum by modulating the local release of dopamine through nicotinic receptors, independently of distant somatic spiking (Threlfell et al., 2012). Thus, Chls sit in a strategic position to orchestrate the events underlying corticostriatal plasticity onto SPNs (Calabresi et al., 2000; Deffains and Bergman, 2015). In this manner, the PT $\rightarrow$ Chls $\rightarrow$ glutA $\rightarrow$ SPNs motif described here may provide a circuit mechanism supporting the delivery of a permissive signal for corticostriatal plasticity onto action-specific SPNs (Deffains and Bergman, 2015), conveyed by temporo-spatially restricted changes in striatal levels of acetylcholine, glutamate and maybe dopamine when movement is executed. In fact, the activation of Chls silence neighboring Chls by feed-back inhibition, further coordinating spatiotemporal acetylcholine fluctuations (Sullivan et al., 2008; Dorst et al., 2020). Therefore, our work supports a model where PT long-range axons, besides directly selecting or invigorating the execution of a specific action by recruiting SPNs encoding for that action, could also gate plastic changes in corticostriatal synapses onto those SPNs by selectively activating specific Chls. happens because the depolarizations evoked by the activation PT inputs alone do not reach spiking threshold in SPNs. Dissimilar expression levels of ChR2 between the transgenic lines could account for this divergence. This technical limitation could coexist with, and it is hard to distinguish from, differences in the IT/PT connectivity strength due to biological constrains that are technically challenging to measure, like, for example, the absolute number of afferents that an individual SPNs sample from each pathway. Another explanation could be the location and distribution of synaptic inputs onto the dendrites of SPNs. It is well established that SPNs need to transition from down- to up-states before spiking (O'Donnell and Grace, 1995; Wilson and Kawaguchi, 1996). It is also clear that up-states require a precise spatio-temporal coordination of synaptic inputs to occur, with a minimum number of clustered inputs coactivated in a distal dendritic fragment (Plotkin et al., 2011). Thus, one possibility is that IT, but not PT, inputs are arranged onto SPNs in a configuration that could trigger state transitions by themselves. Maybe, in low-magnesium and high-calcium ACSF, boosted 
PT $\rightarrow$ Chl activation triggers acetylcholine-dependent glutamate release from different long-range axons innervating SPNs, including IT inputs (Figure 5K), with a spatial arrangement that facilitates spiking. Future experiments mapping the organization of corticostriatal inputs onto SPNs will help further elucidating this issue.

In conclusion, our work dissects, for the first time, the IT and PT corticostriatal connectivity to Chls, uncovering a circuit motif that elicits Chls activation upon PT putative motor command signals. Altogether, our results propose a model where PT long-range axons, besides directly selecting or invigorating the execution of a specific action by recruiting SPNs encoding for that action, gate corticostriatal plasticity in specific synapses onto those SPNs. Therefore, the results presented here provide new insights on the polysynaptic impact of corticostriatal IT and PT signals onto SPNs, with potential implications for movement, plasticity and learning. Further studies investigating how other striatal microcircuit players sample and integrate long-range IT and PT inputs, as well as pathwayspecific roles in vivo, will help tackle these complex corticostriatal circuits underlying motor learning and behavior. and A. Klaus for comments on the manuscript and members of the Costa Lab for useful discussion. This work was supported by a fellowship/contract from Fundação para a Ciência e a Tecnologia to Champalimaud Foundation. 
N.A.M. designed, performed and analyzed the experiments with input from I.I. and R.M.C. A.F.I. did

472 Figure Legends

Figure 1. PT corticostriatal inputs evoke biphasic responses onto SPNs.

(A, B) 10x confocal image tiles of coronal brain slices from IT-ChR2-EYFP (A) or PT-ChR2-EYFP (B) mice. callosum. Cx: cortex.

(C) Diagram of experimental design: SPNs from the DLS of IT- or PT-ChR2-EYFP expressing mouse lines axons with identical illumination power but different stimulus duration, inter-stimulus interval: $30 \mathrm{~s}$. Scale bar: $20 \mathrm{~ms}, 100 \mathrm{pA}$.

(E) Total charge of the EPSC as a function of the amplitude of its $1^{\text {st }}$ peak. Each point represents an individual trial. $n=423$ trials from 26 SPNs from 10 IT-ChR2-EYFP mice (blue). $n=797$ trials from 34 SPNs from 19 PT-ChR2-EYFP mice (red). Solid lines are a linear fit for each group. Slope with 95\% confidence 

(blue) or PT (red) axons. Data is binned in 8 bins of 50 pA. the $1^{\text {st }}$ peak. Data is binned in 4 bins of 100 pA. Datapoints are mean \pm SEM. (red) axons. Data is binned in 5 bins of $5 \mathrm{~ms}$. and PT-ChR2-EYFP (K) mice. IT: $n=423$ trials from 26 SPNs from 10 mice. PT: $n=797$ trials from 34 SPNs from 19 mice. Inset in K: histogram showing the latencies from the $1^{\text {st }}$ peak to the $2^{\text {nd }}$ peak in the same trial. $n=610$ trials from 34 SPNs from 19 PT-ChR2-EYFP mice. point represents an individual neuron. $n=26$ SPNs from 10 IT-ChR2-EYFP mice (blue). $n=34$ SPNs from 19 PT-ChR2-EYFP mice (red).

(M) Example traces from individual SPNs showing $1^{\text {st }}$ peaks of different amplitudes when photostimulating IT (blue) or PT (red) fibers. Light gray traces are the five individual trials corresponding to the blue/red average traces. Scale bar: $20 \mathrm{~ms}, 100 \mathrm{pA}$.

(N) Probability of evoking a $2^{\text {nd }}$ peak upon photostimulation of IT (blue) or PT (red) fibers, for individual neurons. $n=26$ SPNs from 10 IT-ChR2-EYFP mice. $n=34$ SPNs from 19 PT-ChR2-EYFP mice. hChR2(H134R)-EYFP-WPRE-pA in the motor cortex (M1). Five weeks later, SPN EPSCs were recorded upon wide-field photostimulation of M1 $\rightarrow$ DLS PT fibers with LED blue light.

(P) Example traces from a SPN when photostimulating M1 $\rightarrow$ DLS PT fibers with identical power but bar: $20 \mathrm{~ms}, 50 \mathrm{pA}$. 
Figure 2. Corticostriatal PT $\rightarrow$ SPN EPSC second peak is mediated by striatal Chls.

513 (A) Left, EPSCs from a representative SPN when identically photostimulating PT fibers in ACSF (red) or

514 PTX (gray). Thin light gray traces are the five individual trials corresponding to the thicker mean traces.

515 Scale bar: 20 ms, 50 pA. Right, mean traces normalized to the first peak.

516 (B) From top to bottom: plots showing the amplitude of the $1^{\text {st }}$ peak of the EPSCs, the charge ratio, 517 the peak ratio and the response probability for a $2^{\text {nd }}$ peak as a function of time, for the whole experiment in A. Data points are individual trials elicited every $30 \mathrm{~s}$ (for charge ratio and peak ratio, filled circles are EPSCs with a detected $2^{\text {nd }}$ peak). Response probability was calculated within a moving window of 3 consecutive trials. Shadowed areas highlight the individual trials (thin light gray traces in A) averaged for each condition in A (red: ACSF; gray: PTX). $n=8$ SPNs from 5 PT-ChR2-EYFP mice. Bars represent mean. $p=0.54688$ (C); $p=0.0078125$ (D); Wilcoxon signed-rank test.

(E) Left, EPSCs from a representative SPN when identically photostimulating PT fibers in ACSF (red) or MLA (black). Thin light gray traces are the five individual trials corresponding to the thicker mean traces. Scale bar: 20 ms, 50 pA. Right, mean traces normalized to the first peak. peak ratio and the response probability for a $2^{\text {nd }}$ peak as a function of time, for the whole experiment in E. Data points are individual trials elicited every $30 \mathrm{~s}$ (for charge ratio and peak ratio, circles: EPSC with $2^{\text {nd }}$ peak; crosses: EPSC without $2^{\text {nd }}$ peak). Response probability was calculated within a moving window of 3 consecutive trials. Shadowed areas highlight the individual trials (thin light gray traces in E) averaged for each condition in E (red: ACSF; black: MLA). 
$534(G, H)$ Charge ratio $(G)$ and peak ratio $(H)$ for individual SPNs in ACSF (red) and MLA (black) conditions. $n=10$ SPNs from 7 PT-ChR2-EYFP mice. Bars represent mean. $p=0.0019531(G) ; p=0.0039063(H)$; Wilcoxon signed-rank test. EYFP mice. Bars represent mean. $p=0.00054847(\mathrm{I}) ; \mathrm{p}=0.0030623$ (J); Wilcoxon rank sum test. and after the addition of PTX (gray, K) or MLA (black, L). PTX, n=8 SPNs from 5 PT-ChR2-EYFP mice; MLA, $n=10$ SPNs from 7 PT-ChR2-EYFP mice.

Figure 3. PT neurons provide stronger relative inputs to Chls than IT neurons.

545 (A) Diagram of experimental design. Left: first, a Chl from the DLS was recorded while IT- or PT-ChR2 ${ }^{+}$ presynaptic terminals were photostimulated following a $3 \times 4$ grid pattern using a 1-photon blue laser in the presence of TTX and 4-AP. Right: second, a neighboring SPN $(<100 \mu \mathrm{m}$ apart $)$ was recorded while photostimulating with the same grid pattern at identical illumination conditions.

(B) $25 x$ confocal image showing a pair of Chl (magenta arrowhead) and SPN (black arrowhead) in the DLS of an IT-ChR2-EYFP mouse filled with biocytin during the recordings and revealed with streptavidin. Scale bar: $100 \mu \mathrm{m}$. cc: corpus callosum. Cx: cortex.

552 (C) Top: Input-output curve showing the firing pattern of the Chl in B, scale bar: $20 \mathrm{mV}, 500 \mathrm{~ms}$. Gray traces are current injection steps; scale bar: 100 pA. Bottom: 20x single confocal planes showing the neuronal body of the Chl in B (left, magenta), its immunoreactivity to anti-ChAT antibodies (center, cyan) and the overlap of both signals (right). Scale bar: $10 \mu \mathrm{m}$. 
traces). Right, reconstruction of the neuronal morphology from the SPN in B with the relative position of its photostimulation grid (blue circles) and its correspondent $3 \times 4$ matrix of recorded EPSCs (black traces). Left scale bar: $100 \mu \mathrm{m}$. Right scale bar: $20 \mathrm{~ms}, 50$ pA.

(E) Comparison of total input charge transferred to pairs of neighboring Chls and SPNs when activating represent mean. $\mathrm{p}=0.16016 ; \mathrm{n}=10$ pairs from 9 mice; Wilcoxon signed-rank test.

(F) Total input to ChI-SPN pairs normalized to the total input to the SPN, when activating PT fibers. 9 mice.

(G) Comparison of total input charge transferred to pairs of neighboring Chls and SPNs when activating represent mean. $p=0.0039063 ; n=10$ pairs from 10 mice; Wilcoxon signed-rank test. Bars represent median. IT $\rightarrow$ SPN vs. IT $\rightarrow$ Chl, $p=0.0039063$; Wilcoxon signed-rank test. $n=10$ pairs from 10 mice. mice. Horizontal black line: median; filled black circle: mean; box edges: $25^{\text {th }}$ and $75^{\text {th }}$ percentile; whiskers: maximum and minimum excluding outliers; crosses: outliers.

Figure 4. ChIs indirectly excite SPNs via an acetylcholine-dependent glutamate release mechanism. 

when photostimulating Chls (magenta). n=379 trials from 20 SPNs from 9 ChAT-ChR2-EYFP mice. For comparison, the distribution of the latencies to the $1^{\text {st }}$ (dark gray) or $2^{\text {nd }}$ peak (light gray) in PT-ChR2EYFP mice (same data than Figure $1 \mathrm{~K}$ ) is shown.

(C) Top: example of an individual experiment showing EPSCS from a SPN when identically photostimulating ChAT-ChR2-EYFP neurons before (ACSF, magenta) and after the application of MLA (black). Thin light gray traces are individual trials corresponding to the thicker mean traces. Same scale as in D. Bottom: plots showing the EPSC charge and peak amplitude as a function of time, for the same experiment of the traces above. Black filled circles represent individual trials elicited every $30 \mathrm{~s}$. Shadowed areas highlight the individual trials (thin light gray traces above) averaged for each condition (magenta: ACSF; dark gray: MLA).

(D) Top: example of an individual experiment showing EPSCs from a SPN when identically photostimulating ChAT-ChR2-EYFP neurons before (ACSF, magenta) and after the application of DNQX and APV (gray). Thin light gray traces are individual trials corresponding to the thicker mean traces. Scale bar: $25 \mathrm{~ms}, 50 \mathrm{pA}$. Bottom: plots showing the EPSC charge and peak amplitude as a function of time, for the same experiment of the traces above. Black filled circles represent individual trials 597 elicited every $30 \mathrm{~s}$. Shadowed areas highlight the individual trials averaged for each condition 598 (magenta: ACSF; light gray: DNQX and APV).

599 (E, F) EPSC charge (E) and amplitude (F) for individual SPNs in ACSF (magenta) and MLA (black) 600 conditions. $n=10$ SPNs from 5 ChAT-ChR2-EYFP mice. Bars represent mean. $p=0.0019531$ (E); $\mathrm{p}=0.0039063(\mathrm{~F})$; Wilcoxon signed-rank test. $\mathrm{p}=0.0078125(\mathrm{H})$; Wilcoxon signed-rank test. 
(A) Total area of the EPSP evoked upon photostimulation of corticostriatal IT (blue) or PT (red) fibers individual trial. $n=286$ trials from 11 SPNs from 4 IT-ChR2-EYFP mice (blue); $n=384$ trials from 11 SPNs from 4 PT-ChR2-EYFP mice (red). Solid lines are a linear fit for each group. Slope with 95\% Cl: IT, 26.47 $\mathrm{pC} / \mathrm{mV}^{*} \mathrm{~ms}(25.95,26.99) ; \mathrm{PT}, 37.05 \mathrm{pC} / \mathrm{mV}^{*} \mathrm{~ms}(36.07,38.03)$. Intersect with $95 \% \mathrm{Cl}: \mathrm{IT},-7.47 \mathrm{pC}$ ($17.11,2.17) ; \mathrm{PT},-8.68 \mathrm{pC}(-17.83,0.47)$.

(B) Total area of the mean EPSP for each SPN as a function of the amplitude of its $1^{\text {st }}$ peak. Each point traces are the individual trials underlying the blue/red mean traces. Scale bar: $25 \mathrm{~ms}, 5 \mathrm{mV}$. individual neurons. $\mathrm{n}=11$ SPNs from 4 IT-ChR2-EYFP mice. $\mathrm{n}=11$ SPNs from 4 PT-ChR2-EYFP mice.

(E) Spike probability as a function of photostimulation duration recorded from SPNs when activating IT (blue) or PT (red) fibers in normal ACSF (solid line) or ACSF with $0 \mathrm{mM} \mathrm{Mg}^{2+}$ and $4 \mathrm{mM} \mathrm{Ca}^{2+}$ (dotted line). Datapoints are mean \pm SEM. recorded in ACSF with $0 \mathrm{mM} \mathrm{Mg}^{2+}$ and $4 \mathrm{mM} \mathrm{Ca}^{2+} . \mathrm{p}=0.2975(\mathrm{~F}) ; \mathrm{p}=2.28 \times 10^{-11}(\mathrm{G})$; Wilcoxon rank sum test. IT: $n=38$ spikes from 13 SPNs from 11 mice. PT: $n=52$ spikes from 7 SPNs from 7 mice. 
(I) Example traces recorded from the same SPN when photostimulating IT fibers with increasing stimulus duration in ACSF with $0 \mathrm{mM} \mathrm{Mg}^{2+}$ and $4 \mathrm{mM} \mathrm{Ca}^{2+}$. Same scale as in J. stimulus duration in ACSF with $0 \mathrm{mM} \mathrm{Mg}^{2+}$ and $4 \mathrm{mM} \mathrm{Ca}^{2+}$. Scale bar: $25 \mathrm{~ms}, 20 \mathrm{mV}$. cortical neurons project to both SPNs (1) and Chls (2). While PT $\rightarrow$ SPNs (1, red) and PT $\rightarrow$ Chls $(2$, red) input strength is similar, IT $\rightarrow$ Chls (2, blue) connection is weaker than IT $\rightarrow$ SPNs (1, blue). Within the striatum, Chls convey excitation to SPNs by recruiting long-range glutamatergic terminals reaching DLS (3 and inset). Ach, acetylcholine; nAchR, nicotinic receptors; Glut, glutamate. Right, schematic of the activation of the different circuit players upon IT or PT photostimulation and its impact on the recorded SPNs. Magenta and black horizontal lines represent Chls and SPNs spiking threshold, respectively. by the Lead Contact, Rui M. Costa (rc3031@columbia.edu). 
All procedures followed the Champalimaud Center for the Unknown Ethics committee guidelines, approved by the Portuguese Veterinary General Board (Ref. No. 0421/000/000/2014). Both male and female transgenic mice ranging from 40 to 76 days of age were used. Mice were allocated to their experimental groups according to their genotype and age, so the age of the recorded neurons from IT and PT cohorts at each experimental condition resulted balanced (Supplemental Table 1). Animals were group-housed on a $12 \mathrm{hr}$ light/dark cycle with ad libitum access to food and water. IT-cre (T/x3 http://www.gensat.org/), PT-cre (OE25 line, STOCK Tg(Chrna2-cre)OE25Gsat/Mmucd, RRID:MMRRC_036502-UCD, GENSAT) and ChAT-Cre (B6;129S6 chattm2(cre)Lowl/J, Jackson Laboratory, \#006410) were crossed with a cre-dependent ChR2-EYFP line (Ai32, B6;129S-Gt(ROSA)26Sor ${ }^{\text {tm32(CAG- }}$ COP4*H134R/EYFP)Hze/J, Jackson Laboratory, \#012569) generating double transgenic lines expressing ChR2EYFP in specific neuronal populations. In some cases, triple transgenic mice were used by crossing ITor PT-cre lines with Ai32 line and a transgenic BAC Drd1a-tdTomato line (B6.Cg-Tg(Drd1atdTomato)6Calak/J, Jackson Laboratory, \#016204). All lines were on C57BL/6 background by backcrossing with $\mathrm{C} 57 \mathrm{BL} / 6 \mathrm{~J}$ inbred mice for at least 8 generations. 
675 UCD, were obtained from the Mutant Mouse Resource and Research Center (MMRRC) at University 676 of California at Davis, an NIH-funded strain repository, and was donated to the MMRRC by Nathaniel 677 Heintz, Ph.D., The Rockefeller University, GENSAT and Charles Gerfen, Ph.D., National Institutes of Health, National Institute of Mental Health.

\section{Slice preparation}

Mice from 40 to 76 days of age were decapitated after deeply anesthetized with isoflurane. Brains were then dissected in ice-chilled choline chloride solution ( $110 \mathrm{mM}$ choline chloride, $25 \mathrm{mM} \mathrm{NaHCO}$, $25 \mathrm{mM}$ d-glucose, $11.6 \mathrm{mM}$ sodium ascorbate, $7 \mathrm{mM} \mathrm{MgCl}$, $3.1 \mathrm{mM}$ sodium pyruvate, $2.5 \mathrm{mM} \mathrm{KCl}$, $1.25 \mathrm{mM} \mathrm{NaH} 2 \mathrm{PO} 4$ and $0.5 \mathrm{mM} \mathrm{CaCl} 2$, bubbled with 95\% O2/5\% CO2) and coronal slices were cut (300 $\mu \mathrm{m}$ thickness) using a Leica VT1200S vibratome. Slices were incubated at 37으 in ACSF (127 mM $\mathrm{NaCl}, 25 \mathrm{mM}$ NaHCO3, $25 \mathrm{mM}$ d-glucose, $2.5 \mathrm{mM} \mathrm{KCl}, 2 \mathrm{mM} \mathrm{CaCl} 2,1 \mathrm{mM} \mathrm{MgCl} 2$ and $1.25 \mathrm{mM}$ $\mathrm{NaH} 2 \mathrm{PO} 4$, bubbled with $95 \% \mathrm{O} 2 / 5 \% \mathrm{CO} 2$ ) for 30 minutes before starting recording.

\section{Electrophysiology and photostimulation}

Data was recorded using a Multiclamp 700B amplifier (Molecular Devices), digitized with a Digidata 1440 (Molecular Devices), and acquired at $10 \mathrm{kHz}$ with pClamp 10 software (Molecular Devices). 
low-magnesium/high-calcium ACSF, the internal solution was supplemented with Fluo-4 $(200 \mu \mathrm{M}$, Invitrogen) for other imaging purposes. All recordings were performed in heated (37으) normal ACSF or low-magnesium/high-calcium ACSF (127 mM NaCl, 25 mM NaHCO3, 25 mM d-glucose, 2.5 mM KCl, $4 \mathrm{mM} \mathrm{CaCl} 2$ and $1.25 \mathrm{mM} \mathrm{NaH} 2 \mathrm{PO} 4)$ perfused at $1.5-2 \mathrm{~mL} / \mathrm{min}$ rate. In voltage-clamp experiments, SPNs and Chls were clamped at $-80 \mathrm{mV}$ and $-55 \mathrm{mV}$, respectively. All recorded neurons were at least at $40 \mu \mathrm{m}$ depth from the slice surface. For each photostimulation trial, input resistance was monitored with a hyperpolarizing test pulse. After each experiment, SPNs identity was confirmed by imaging their morphology and dendritic spines (filled with Alexa 594, Supplemental Figure 1A) at 60x with a BX61WI Olympus microscope, with galvanometer-based scanning system (Bruker) and a 2-photon Ti:sapphire laser ( $820 \mathrm{~nm}$ for imaging Alexa 594, Coherent), controlled by PrairieView software (Bruker). Somatic area and distance between Chls and SPNs within the same neuronal pair were measured from these images using ImageJ/Fiji (NIH). Chls resting membrane potential was measured at break-in. Metrics from Chls spikes (inter-spike interval and half-with) and sag were extracted from current-clamp recordings by running a set of 15 square current steps ( 1 s duration, $20 \mathrm{pA}$ increase, starting at -160 pA) before bath application of 4-AP and TTX. The position of the recorded neurons in the DLS was visually confirmed at 10x. All neurons were recorded from left DLS.

Wide-field photostimulation was performed using two fiber-coupled $\sim 460 \mathrm{~nm}$ LEDs (Doric lenses) attached to the $60 x$ microscope objective ( $90 \circ$ apart from each other), to standardize the distance from the light source to the slice across experiments. In order to maximize the amount of light reaching the slice below the objective, the intensity of the illumination was fixed at maximum power ( $\sim 10$ and 14 $\mathrm{mW}$ for each LED) with a SLC-SA/SV/AA/AV series LED controller (Mightex, Canada). Using PrairieView software, the light pulse duration was varied to elicit responses spanning the range of EPSC/P amplitudes explored. For laser-photostimulation of inputs to SPN-Chl pairs, light was delivered using a Point-Photoactivation module (Bruker) coupled to a 1-photon $473 \mathrm{~nm}$ laser (Coherent). The grid was positioned so the soma of the recorded neuron located in the center of a horizontal line 
photostimulated twice, following a non-neighboring pattern, maximizing the distance between consecutive laser pulses and resulting in an interval of $>6$ min between illuminations in the same location (inter-location interval: $30 \mathrm{~s}$; inter-grid repetition interval: $1 \mathrm{~min}$ ). At the beginning of each grid experiment, laser intensity and duration were calibrated with PrairieView software so that the total input charge for IT $\rightarrow$ Chls and PT $\rightarrow$ Chls EPSCs were comparable (Results and Figure 3E and G). in normal ACSF with PTX and using cesium-based internal solution (132 mM Cs-gluconate, $10 \mathrm{mM}$ sodium phosphocreatine, $10 \mathrm{mM}$ HEPES, $3 \mathrm{mM}$ sodium l-ascorbate, $4 \mathrm{mM} \mathrm{MgCl} 2,4 \mathrm{mM}$ Na2ATP, 0.4 mM Na2GTP).

\section{Data analysis}

Electrophysiology traces were analyzed using Matlab (MathWorks). Total EPSC/P charge/area was calculated as the integral of the trace in a window of $65 \mathrm{~ms}$ starting with photostimulation. EPSC/P $1^{\text {st }}$ peak amplitude was computed as the minimum (EPSC) / maximum (EPSP) value in a window between the start of illumination and $35 \mathrm{~ms}$. To minimize confounding EPSC/P $2^{\text {nd }}$ peak with $1^{\text {st }}$ peak in cases where the $2^{\text {nd }}$ peak had higher absolute amplitude than the $1^{\text {st }}$ peak, the interval from the photostimulation until the detected peak was scrutinized. When an earlier a peak (with amplitude > $25 \%$ of the detected peak) followed by a valley (with depth $>5 \%$ of the detected peak) was present, the earlier value was counted as the EPSC/P $1^{\text {st }}$.

The decay of the EPSC/P $1^{\text {st }}$ phase was subtracted from the EPSC $2^{\text {nd }}$ phase by subtracting a two-term exponential model from the recorded trace. In order to exclude the datapoints corresponding to the detection window of the EPSC/P $2^{\text {nd }}$ phase from the model fit, we used data from two discontinuous periods ( $50 \mathrm{~ms}$ in total). We defined an initial $10 \mathrm{~ms}$ period, starting where the EPSC/P $1^{\text {st }}$ peak decayed $10 \%$ of its amplitude and finishing with the start of the detection window, and a second 40 ms period 
beginning immediately after that window. Thus, the $2^{\text {nd }}$ phase detection period was restricted to a fixed-size window spanning from 10 to $32 \mathrm{~ms}$ after the EPSC/P $1^{\text {st }}$ peak decayed to $90 \%$ of its amplitude. Within this window, EPSC/P $2^{\text {nd }}$ phase peak amplitude and charge/area were computed as the minimum (EPSC) / maximum (EPSP) value and the integral of the subtracted trace, respectively. EPSC/P $2^{\text {nd }}$ phase was detected when the subtracted trace crossed a negative (EPSC) / positive (EPSP) phase charge was calculated by subtracting the EPSC $2^{\text {nd }}$ phase charge from the total EPSC charge. Charge ratio was calculated by dividing the charge of the EPSC $2^{\text {nd }}$ phase over the total EPSC charge. Peak ratio was computed by dividing the EPSC $2^{\text {nd }}$ peak amplitude over the EPSC $1^{\text {st }}$ peak amplitude. In pharmacological experiments, baseline conditions were computed once the amplitude of the EPSC first peak stabilized after an initial ramping up period (Supplemental Figure 2). In Supplemental Figure 2A, peak amplitude was normalized to the amplitude of the first trial while charge ratio and peak ratio were normalized to the first detected EPSC $2^{\text {nd }}$ phase. trial-by-trial and neuron-based analyses used the same analysis criteria. Response probability was calculated by dividing the number of detected second phases by the total number of photostimulations in the bin (trial-by trial, Figure $\mathbf{1 F}$ and I) or SPNs (neuron-based, Figure $\mathbf{1 N}$ and 5D). Overall spike latency was computed from the start of photostimulation to the peak of the action potential. This period was then sub-divided into early and late sub-periods using the start of the EPSP (detected when the trace crossed a positive threshold of 3-fold the standard deviation of baseline period), as the border between them. Population spike probability and population spike latency were first calculated for individual neurons (number of spikes/total number of trials in the same light condition, and mean latency to spike in the same light condition, respectively) and then averaged across neurons within the same illumination condition. For spike analysis, only traces with baseline membrane potential below (or equal to) $-60 \mathrm{mV}$ were included. 
Modulation index was calculated using the formula: ( $X$ in ACSF $-X$ in Drug) / ( $X$ in ACSF $+X$ in Drug). or EPSC $1^{\text {st }}$ or $2^{\text {nd }}$ phase peak amplitude.

For ChAT-ChR2 experiments, EPSC charge was calculated as the integral of the trace in a window of 65 ms starting with photostimulation. EPSC peak amplitude was computed as the minimum value in a window between the start of illumination and $60 \mathrm{~ms}$. Latencies to EPSC peak were counted from the start of the illumination pulse. with the light pulse. ChI/SPN input ratio was calculated by dividing the sum of all EPSC charges in the Chl over the sum of all EPSC charges in its paired SPN. Chls sag difference was calculated as the difference between the mean membrane potential from $5 \mathrm{~ms}$ at the beginning and $5 \mathrm{~ms}$ at the end (800 ms apart) of a 1 s hyperpolarizing current step of -160 pA.

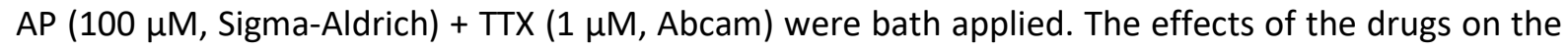
responses were tested after 5 minutes of drug recirculation in the recording chamber. From the 10 MLA experiments in Figure 2, in 8 cases MLA was added alone; in 2 cases MLA was added together with the nicotinic blocker Mecamylamine (MEC, $100 \mu \mathrm{M}$, Sigma-Aldrich). Monosynaptic connectivity was tested after, at least, 10 minutes of 4-AP and TTX recirculation. 
IT- or PT-ChR2-EYFP mice at 60 days of age were intracardiacally perfused with $4 \%$ paraformaldehyde. Brains were removed and coronally sliced (50 $\mu \mathrm{m}$ thickness). Slices were mounted using Mowiol and imaged with a Zeiss LSM 710 confocal microscope.

Slices with biocytin-filled Chl-SPN pairs were fixed in 4\% paraformaldehyde for at least two hours at Mowiol and imaged using a Zeiss LSM 710 confocal microscope. Colocalization of Streptavidin and ChAT was assessed using stacks of individual confocal planes at 20x. Dendritic morphology was reconstructed and measured from tiles of 25x images using neuTube (Feng et al., 2015) and Simple Neurite Tracer (Longair et al., 2011) plugin from ImageJ/Fiji (NIH). Reconstruction traces in Figure 3 were exported using the HBP Neuron Morphology Viewer (Bakker et al., 2017).

\section{Stereotaxic surgery}

817 For viral injection in Figure 10-P, a female PT-cre mouse of 24 days was anesthetized with isoflurane

$818(1 \%-3 \%$, plus oxygen at $1-1.5 \mathrm{l} / \mathrm{min})$ and head-fixed using a stereotaxic frame (David Kopf Instruments, 819 Model 962LS) over a heating pad (ATC1000, World Precision Instruments) at 35-37으. A small craniotomy was drilled over the left M1 following the coordinates (from bregma): $0.55 \mathrm{~mm}$ anterior / 
was then lowered until reaching $750 \mu \mathrm{m}$ of depth from the brain surface. $300 \mathrm{~nL}$ of AAV5-EF1a-DIO-

\section{QUANTIFICATION AND STATISTICAL ANALYSIS}

830 All statistical analyses were done with Matlab (MathWorks). Since normality was not assumed or rejected using Kolmogorov-Smirnov test, non-parametric two-tailed Wilcoxon rank sum test or twotailed Wilcoxon signed-rank test were used across the manuscript for independent and paired comparisons, respectively. Statistical significance was defined as: ns, Non-significant, ${ }^{*} p<0.05,{ }^{* *} p$ $<0.01, * * * p<0.001$ and ${ }^{* * * *} p<0.0001$. Sample size were not predetermined but our groups are in line with previous studies (Tanimura et al., 2019). Sample size, specific statistical test, exact $p$ value and additional information are detailed in the figure legends or Results. Data is presented as mean \pm standard error of the mean (SEM).

\section{KEY RESOURCES TABLE}

\begin{tabular}{|l|l|l|}
\hline REAGENT or RESOURCE & SOURCE & IDENTIFIER \\
\hline Antibodies & \multicolumn{2}{l|}{} \\
\hline Goat anti-ChAT primary antibody & Chemicon/Millipore & Cat\#AB144P \\
\hline Donkey anti-goat Alexa-405 conjugated secondary antibody & Abcam & Cat\#ab175664 \\
\hline Streptavidin conjugated with Alexa-594 & Life Technologies & Cat\#16892 \\
\hline Bacterial and Virus Strains & & \\
\hline AAV5-EF1a-DIO-hChR2(H134R)-EYFP & $\begin{array}{l}\text { University of North } \\
\text { Carolina Vector Core }\end{array}$ & Cat\#20298 \\
\hline
\end{tabular}




\begin{tabular}{|c|c|c|}
\hline \multicolumn{3}{|l|}{ Chemicals, Peptides, and Recombinant Proteins } \\
\hline Alexa Fluor ${ }^{\mathrm{TM}} 594$ dye & Molecular probes & Cat\#A10438 \\
\hline Biocytin & Sigma-Aldrich & $\begin{array}{l}\text { Cat\#B4261; CAS: } 576- \\
19-2\end{array}$ \\
\hline Picrotoxin (PTX) & Tocris & $\begin{array}{l}\text { Cat\#1128; CAS: } 124- \\
87-8\end{array}$ \\
\hline Methyllycaconitine citrate salt (MLA) & Sigma-Aldrich & $\begin{array}{l}\text { Cat\#M168; CAS: } \\
112825-05-5\end{array}$ \\
\hline 6,7-Dinitroquinoxaline-2,3-dione disodium salt (DNQX) & Tocris & $\begin{array}{l}\text { Cat\#2312; CAS: } \\
\text { 1312992-24-7 }\end{array}$ \\
\hline DL-2-Amino-5-phosphonopentanoic acid sodium salt (APV) & Tocris & $\begin{array}{l}\text { Cat\#3693; CAS: } \\
\text { 1303993-72-7 }\end{array}$ \\
\hline Tetradotoxine citrate (TTX) & Abcam & $\begin{array}{l}\text { Cat\#ab120055; CAS: } \\
18660-81-6\end{array}$ \\
\hline 4-Aminopiridine (4-AP) & Sigma-Aldrich & $\begin{array}{l}\text { Cat\#A78403; CAS: } \\
\text { 504-24-5 }\end{array}$ \\
\hline Mecamylamine hydrochloride (MEC) & Sigma-Aldrich & $\begin{array}{l}\text { Cat\#M9020; CAS: 826- } \\
39-1\end{array}$ \\
\hline \multicolumn{3}{|l|}{ Experimental Models: Organisms/Strains } \\
\hline Tg(TIx3-cre)PL58Gsat/Mmucd (TIx3, IT-cre) & GENSAT & $\begin{array}{l}\text { RRID:MMRRC_03667 } \\
\text { 0-UCD }\end{array}$ \\
\hline Tg(Chrna2-cre)OE25Gsat/Mmucd (OE25, PT-cre) & GENSAT & $\begin{array}{l}\text { RRID:MMRRC_03650 } \\
\text { 2-UCD }\end{array}$ \\
\hline B6;129S6Chattm2(cre)Lowl/J (ChaT-cre) & The Jackson Laboratory & Cat\#006410 \\
\hline 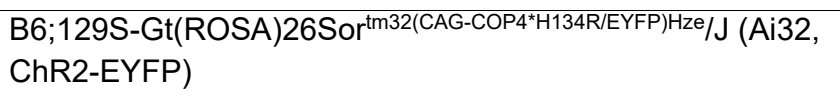 & The Jackson Laboratory & Cat \#012569 \\
\hline B6.Cg-Tg(Drd1a-tdTomato)6Calak/J & The Jackson Laboratory & Cat \#016204 \\
\hline \multicolumn{3}{|l|}{ Software and Algorithms } \\
\hline pClamp 10 & Molecular Devices & $\begin{array}{l}\text { http://www.molecularde } \\
\text { vices.com/products/sof } \\
\text { tware/pclamp.html /; } \\
\text { RRID:SCR_011323 }\end{array}$ \\
\hline ImageJ/Fiji & $\mathrm{NIH}$ & $\begin{array}{l}\text { https://imagej.net/ ; } \\
\text { RRID:SCR_003070 }\end{array}$ \\
\hline Matlab 2018a & MathWorks & $\begin{array}{l}\frac{\text { http://www.mathworks. }}{\text { com/products/matlab/ }} \\
\text { RRID:SCR_001622 }\end{array}$ \\
\hline neuTube & Feng et al. 2015 & $\begin{array}{l}\text { https://www.neutracing. } \\
\text { com/ }\end{array}$ \\
\hline
\end{tabular}




\begin{tabular}{|l|l|l|}
\hline Simple Neurite Tracer & Longair et al. 2011 & $\begin{array}{l}\frac{\text { http://imagej.net/Simpl }}{\text { e Neurite Tracer; }} \\
\text { RRID:SCR_016566 }\end{array}$ \\
\hline HBP Neuron Morphology Viewer & Bakker et al. 2017 & $\begin{array}{l}\text { https://neuroinformatics } \\
\text {.nl/HBP/morphology- } \\
\text { viewer/ }\end{array}$ \\
\hline
\end{tabular}

843 Abudukeyoumu, N., Hernandez-Flores, T., Garcia-Munoz, M. and Arbuthnott, G. W. (2018)

844 'Cholinergic modulation of striatal microcircuits', European Journal of Neuroscience, 49(5), pp. 604622. doi: 10.1111/ejn.13949. in neurosciences, 13(7), pp. 266-271. microcircuits of corticospinal and corticostriatal neurons in motor cortex', Nature Neuroscience, 13(6), pp. 739-744. doi: 10.1038/nn.2538.

Assous, M. and Tepper, J. M. (2018) ‘Excitatory extrinsic afferents to striatal interneurons and interactions with striatal microcircuitry', European Journal of Neuroscience, 49(5), pp. 593-603. doi: 10.1111/ejn.13881.

Bakker, R., García-Amado, M Evangelio, M., Clascá, F. and Tiesinga, P. (2017) ‘P271 Workflow, data format and tools to register neuron morphologies to a reference brain atlas', CNS abstract. 
Brown, S. P. and Hestrin, S. (2009) 'Intracortical circuits of pyramidal neurons reflect their long-range axonal targets', Nature, 457(7233), pp. 1133-1136. doi: 10.1038/nature07658. 2236(99)01501-5.

Campos, F., Alfonso, M. and Durán, R. (2010) 'In vivo modulation of $\alpha 7$ nicotinic receptors on striatal glutamate release induced by anatoxin- $A^{\prime}$, Neurochemistry International, 56(6-7), pp. 850-855. doi: 10.1016/j.neuint.2010.03.010.

Cowan, R. L. and Wilson, C. J. (1994) 'Spontaneous firing patterns and axonal projections of single corticostriatal neurons in the rat medial agranular cortex.', Journal of neurophysiology, 71(1), pp. $17-32$. and excitation across mouse visual cortex depends on the hierarchy of interconnecting areas', eLife, 5(September2016), pp. 1-19. doi: 10.7554/eLife.19332. Segovia, J. (2014) 'A Major External Source of Cholinergic Innervation of the Striatum and Nucleus Accumbens Originates in the Brainstem', Journal of Neuroscience, 34(13), pp. 4509-4518. doi: 10.1523/JNEUROSCI.5071-13.2014. 
plasticity in health and disease', Movement Disorders, 30(8), pp. 1014-1025. doi: $10.1002 / \mathrm{mds} .26300$.

885

Dorst, M. C., Tokarska, A., Zhou, M., Lee, K., Stagkourakis, S., Broberger, C., Masmanidis, S. and

Silberberg, G. (2020) 'Polysynaptic inhibition between striatal cholinergic interneurons shapes their network activity patterns in a dopamine-dependent manner', Nature Communications, 11(1), p. 5113. doi: 10.1038/s41467-020-18882-y.

Economo, M. N. et al. (2018) 'Distinct descending motor cortex pathways and their roles in movement', Nature, 563(7729), pp. 79-84. doi: 10.1038/s41586-018-0642-9. and Koos, T. (2012) ‘GABAergic circuits mediate the reinforcement-related signals of striatal cholinergic interneurons', Nature Neuroscience, 15(1), pp. 123-130. doi: 10.1038/nn.2984. Software Based on the SWC Format', eneuro, 2(1), p. ENEURO.0049-14.2014. doi: 10.1523/ENEURO.0049-14.2014.

Fieblinger, T. et al. (2014) 'Cell type-specific plasticity of striatal projection neurons in parkinsonism and L-DOPA-induced dyskinesia', Nature Communications, 5(1), p. 5316. doi: 10.1038/ncomms6316. striatal matrix: experimental evidence in the squirrel monkey', The Journal of Neuroscience, 13(3), pp. 1120-1137. doi: 10.1523/JNEUROSCI.13-03-01120.1993. the Functional Organization of Cerebral Cortical and Basal Ganglia Circuits', Neuron, 80(6), pp. 13681383. doi: 10.1016/j.neuron.2013.10.016. and HÖkfelt, T. (eds) Handbook of Chemical Neuroanatomy - Volume 12. Elsevier, pp. 371-468. doi: 
Circuitry', in Fryer, A. D., Christopoulos, A., and Nathanson, N. M. (eds) Muscarinic Receptors. Berlin, Heidelberg: Springer Berlin Heidelberg, pp. 223-241. doi: 10.1007/978-3-642-23274-9_10. control', Science, 265(5180), pp. 1826-1831. doi: 10.1126/science.8091209. Neurobiology, 15(6), pp. 638-644. doi: 10.1016/j.conb.2005.10.006.

Gremel, C. M. and Costa, R. M. (2013) 'Orbitofrontal and striatal circuits dynamically encode the shift between goal-directed and habitual actions', Nature Communications, 4(1), p. 2264. doi: $10.1038 /$ ncomms3264. inputs to projection neurons and cholinergic interneurons in the dorsal striatum', PLOS ONE. Edited by B. Arenkiel, 10(4), p. e0123381. doi: 10.1371/journal.pone.0123381. (2015) 'Multimodal Plasticity in Dorsal Striatum While Learning a Lateralized Navigation Task', Journal of Neuroscience, 35(29), pp. 10535-10549. doi: 10.1523/JNEUROSCI.4415-14.2015. 
Hintiryan, H. et al. (2016) 'The mouse cortico-striatal projectome', Nature Neuroscience, 19(8), pp. 1100-1114. doi: 10.1038/nn.4332. 3549. doi: 10.1038/s41467-018-05780-7.

Howe, W. M., Young, D. A., Bekheet, G. and Kozak, R. (2016) 'Nicotinic receptor subtypes International, 100, pp. 30-34. doi: 10.1016/j.neuint.2016.08.009. 30(4), p. 1178-1194.e3. doi: 10.1016/j.celrep.2019.12.095.

Kemp, J. M. and Powell, T. P. (1971) 'The structure of the caudate nucleus of the cat: light and electron microscopy', Philosophical Transactions of the Royal Society of London. B, Biological Sciences, 262(845), pp. 383-401. doi: 10.1098/rstb.1971.0102.

950 Circuits and Self-Paced Action Initiation', Annual Review of Neuroscience, 42(1), pp. 459-483. doi: 10.1146/annurev-neuro-072116-031033. 
Neuron, 60(4), pp. 543-554. doi: 10.1016/j.neuron.2008.11.005.

956

957

958

959

960

961

962

963

964

965

966

967

968

969

970

971

972

973

974

975

976

977

Kress, G. J., Yamawaki, N., Wokosin, D. L., Wickersham, I. R., Shepherd, G. M. G. and Surmeier, D. J.

(2013) 'Convergent cortical innervation of striatal projection neurons', Nature Neuroscience, 16(6), pp. 665-667. doi: 10.1038/nn.3397.

Lee, A. M., Tai, L.-H., Zador, A. and Wilbrecht, L. (2015) 'Between the primate and "reptilian” brain: Rodent models demonstrate the role of corticostriatal circuits in decision making', Neuroscience, 296, pp. 66-74. doi: 10.1016/j.neuroscience.2014.12.042.

Li, N., Chen, T.-W., Guo, Z. V, Gerfen, C. R. and Svoboda, K. (2015) 'A motor cortex circuit for motor planning and movement', Nature, 519(7541), pp. 51-56. doi: 10.1038/nature14178.

Longair, M. H., Baker, D. A. and Armstrong, J. D. (2011) 'Simple neurite tracer: Open source software for reconstruction, visualization and analysis of neuronal processes', Bioinformatics, 27(17), pp. 2453-2454. doi: 10.1093/bioinformatics/btr390.

Mallet, N., Le Moine, C., Charpier, S. and Gonon, F. (2005) ‘Feedforward inhibition of projection neurons by fast-spiking GABA interneurons in the rat striatum in vivo', Journal of Neuroscience, 25(15), pp. 3857-3869. doi: 10.1523/JNEUROSCI.5027-04.2005.

Molnár, Z. and Cheung, A. F. P. (2006) 'Towards the classification of subpopulations of layer V pyramidal projection neurons', Neuroscience Research, 55(2), pp. 105-115. doi: 10.1016/j.neures.2006.02.008.

Morgenstern, N. A., Bourg, J. and Petreanu, L. (2016) 'Multilaminar networks of cortical neurons integrate common inputs from sensory thalamus', Nature Neuroscience, 19(8), pp. 1034-1040. doi: 10.1038/nn.4339.

Morishima, M. (2006) ‘Recurrent Connection Patterns of Corticostriatal Pyramidal Cells in Frontal Cortex', Journal of Neuroscience, 26(16), pp. 4394-4405. doi: 10.1523/JNEUROSCI.0252-06.2006. 

Nelson, A., Abdelmesih, B. and Costa, R. M. (2020) 'Corticospinal neurons encode complex motor signals that are broadcast to dichotomous striatal circuits', bioRxiv, p. 2020.08.31.275180.

Nelson, A. B., Hammack, N., Yang, C. F., Shah, N. M., Seal, R. P. and Kreitzer, A. C. (2014) 'Striatal Cholinergic Interneurons Drive GABA Release from Dopamine Terminals', Neuron, 82(1), pp. 63-70. doi: 10.1016/j.neuron.2014.01.023.

O’Donnell, P. and Grace, A. A. (1995) 'Synaptic interactions among excitatory afferents to nucleus accumbens neurons: Hippocampal gating of prefrontal cortical input', Journal of Neuroscience, 15(5 I), pp. 3622-3639. doi: 10.1523/jneurosci.15-05-03622.1995.

Pennartz, C. M. A., Berke, J. D., Graybiel, A. M., Ito, R., Lansink, C. S., van der Meer, M., Redish, A. D., Smith, K. S. and Voorn, P. (2009) 'Corticostriatal Interactions during Learning, Memory Processing, and Decision Making', Journal of Neuroscience, 29(41), pp. 12831-12838. doi: 10.1523/JNEUROSCI.3177-09.2009.

Petreanu, L., Huber, D., Sobczyk, A. and Svoboda, K. (2007) 'Channelrhodopsin-2-assisted circuit mapping of long-range callosal projections', Nature Neuroscience, 10(5), pp. 663-668. doi: $10.1038 / \mathrm{nn} 1891$.

Petreanu, L., Mao, T., Sternson, S. M. and Svoboda, K. (2009) 'The subcellular organization of neocortical excitatory connections', Nature, 457(7233), pp. 1142-1145. doi: 10.1038/nature07709.

Planert, H., Szydlowski, S. N., Hjorth, J. J. J., Grillner, S. and Silberberg, G. (2010) ‘Dynamics of Synaptic Transmission between Fast-Spiking Interneurons and Striatal Projection Neurons of the Direct and Indirect Pathways', Journal of Neuroscience, 30(9), pp. 3499-3507. doi: 10.1523/JNEUROSCI.5139-09.2010.

Plenz, D. (2003) 'When inhibition goes incognito: feedback interaction between spiny projection neurons in striatal function', Trends in Neurosciences, 26(8), pp. 436-443. doi: 10.1016/S01662236(03)00196-6. 
1002

1003

1004

1005

1006

1007

1008

1009

1010

1011

1012

1013

1014

1015

1016

1017

1018

1019

1020

1021

1022

1023

Plotkin, J. L., Day, M. and Surmeier, D. J. (2011) 'Synaptically driven state transitions in distal dendrites of striatal spiny neurons', Nature Neuroscience, 14(7), pp. 881-888. doi: 10.1038/nn.2848.

Reiner, A. (2010) 'Corticostriatal projection neurons - dichotomous types and dichotomous functions', Frontiers in Neuroanatomy, 4(October), p. 142. doi: 10.3389/fnana.2010.00142.

Shan, Q., Ge, M., Christie, M. J. and Balleine, B. W. (2014) 'The Acquisition of Goal-Directed Actions Generates Opposing Plasticity in Direct and Indirect Pathways in Dorsomedial Striatum', Journal of Neuroscience, 34(28), pp. 9196-9201. doi: 10.1523/JNEUROSCI.0313-14.2014.

Shen, W., Flajolet, M., Greengard, P. and Surmeier, D. J. (2008) ‘Dichotomous Dopaminergic Control of Striatal Synaptic Plasticity', Science, 321(5890), pp. 848-851. doi: 10.1126/science.1160575.

Shen, W., Hamilton, S. E., Nathanson, N. M. and Surmeier, D. J. (2005) 'Cholinergic suppression of KCNQ channel currents enhances excitability of striatal medium spiny neurons', Journal of Neuroscience, 25(32), pp. 7449-7458. doi: 10.1523/JNEUROSCI.1381-05.2005.

Shepherd, G. M. G. (2013) 'Corticostriatal connectivity and its role in disease', Nature Reviews Neuroscience, 14(4), pp. 278-291. doi: 10.1038/nrn3469.

Stephens, B. et al. (2005) 'Evidence of a breakdown of corticostriatal connections in Parkinson's disease', Neuroscience, 132(3), pp. 741-754. doi: 10.1016/j.neuroscience.2005.01.007.

Sullivan, M. A., Chen, H. and Morikawa, H. (2008) 'Recurrent Inhibitory Network among Striatal Cholinergic Interneurons', Journal of Neuroscience, 28(35), pp. 8682-8690. doi: 10.1523/JNEUROSCI.2411-08.2008.

Tanimura, A., Du, Y., Kondapalli, J., Wokosin, D. L. and Surmeier, D. J. (2019) ‘Cholinergic Interneurons Amplify Thalamostriatal Excitation of Striatal Indirect Pathway Neurons in Parkinson's Disease Models', Neuron, 101(3), p. 444-458.e6. doi: 10.1016/j.neuron.2018.12.004.

Tasic, B. et al. (2018) 'Shared and distinct transcriptomic cell types across neocortical areas', Nature, 
563(7729), pp. 72-78. doi: 10.1038/s41586-018-0654-5.

Tepper, J. M., Abercrombie, E. D. and Bolam, J. P. (2007) 'Basal ganglia macrocircuits', in Tepper, J.

M., Abercrombie, E. D., and Bolam, J. P. (eds) Progress in Brain Research - Volume 160. Elsevier, pp. 3-7. doi: 10.1016/S0079-6123(06)60001-0.

Threlfell, S., Lalic, T., Platt, N. J., Jennings, K. A., Deisseroth, K. and Cragg, S. J. (2012) 'Striatal Dopamine Release Is Triggered by Synchronized Activity in Cholinergic Interneurons', Neuron, 75(1), pp. 58-64. doi: 10.1016/j.neuron.2012.04.038.

Wall, N., De La Parra, M., Callaway, E. and Kreitzer, A. (2013) 'Differential Innervation of Direct- and Indirect-Pathway Striatal Projection Neurons', Neuron, 79(2), pp. 347-360. doi: 10.1016/j.neuron.2013.05.014.

Wang, Z., Kai, L., Day, M., Ronesi, J., Yin, H. H., Ding, J., Tkatch, T., Lovinger, D. M. and Surmeier, D. J. (2006) 'Dopaminergic Control of Corticostriatal Long-Term Synaptic Depression in Medium Spiny Neurons Is Mediated by Cholinergic Interneurons', Neuron, 50(3), pp. 443-452. doi: 10.1016/j.neuron.2006.04.010.

Wilson, C. and Kawaguchi, Y. (1996) 'The origins of two-state spontaneous membrane potential fluctuations of neostriatal spiny neurons', The Journal of Neuroscience, 16(7), pp. 2397-2410. doi: 10.1523/JNEUROSCI.16-07-02397.1996.

Wonnacott, S., Kaiser, S., Mogg, A., Soliakov, L. and Jones, I. W. (2000) ‘Presynaptic nicotinic receptors modulating dopamine release in the rat striatum', European Journal of Pharmacology, 393(1-3), pp. 51-58. doi: 10.1016/S0014-2999(00)00005-4.

Yagishita, S., Hayashi-Takagi, A., Ellis-Davies, G. C. R., Urakubo, H., Ishii, S. and Kasai, H. (2014) 'A critical time window for dopamine actions on the structural plasticity of dendritic spines', Science, 345(6204), pp. 1616-1620. doi: 10.1126/science.1255514. 

$10.1016 / 0006-8993(78) 90059-8$.

1054 Yin, H. H., Mulcare, S. P., Hilário, M. R. F., Clouse, E., Holloway, T., Davis, M. I., Hansson, A. C., 1055 Lovinger, D. M. and Costa, R. M. (2009) 'Dynamic reorganization of striatal circuits during the acquisition and consolidation of a skill', Nature Neuroscience, 12(3), pp. 333-341. doi:

1057 $10.1038 / \mathrm{nn} .2261$

1058

Zhou, F.-M., Wilson, C. J. and Dani, J. A. (2002) 'Cholinergic interneuron characteristics and nicotinic properties in the striatum', Journal of Neurobiology, 53(4), pp. 590-605. doi: 10.1002/neu.10150. 

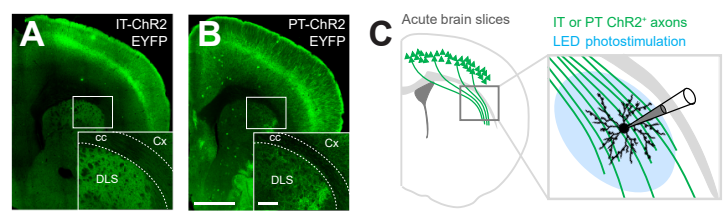

\section{E}

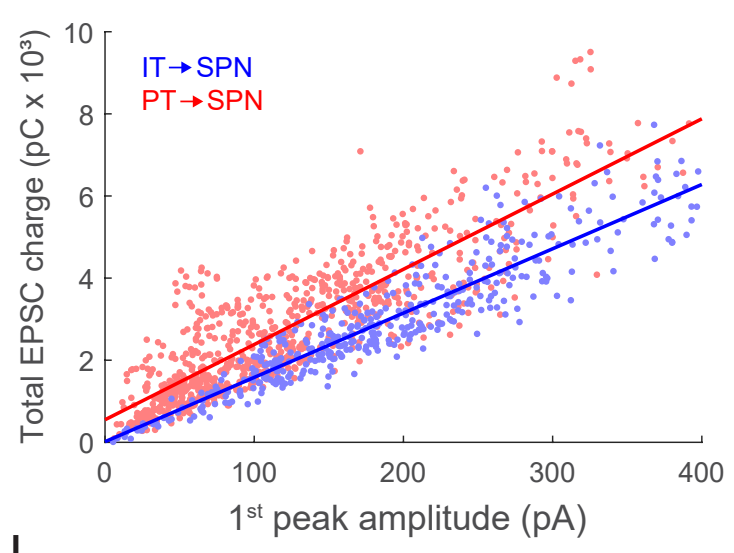

L

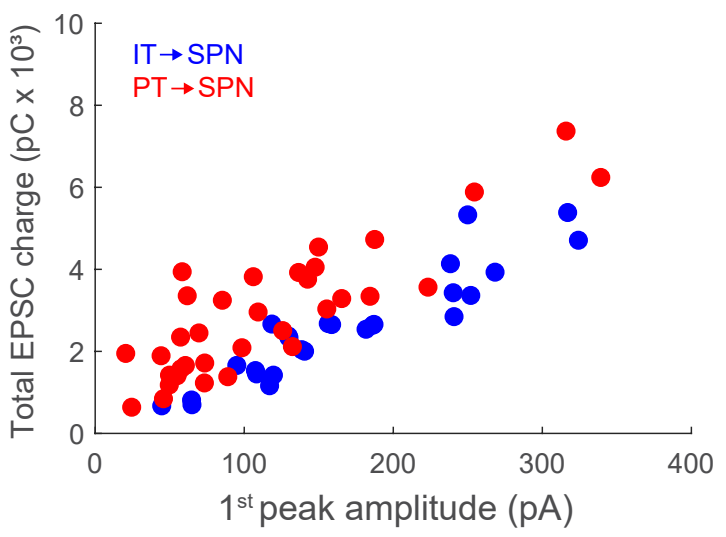

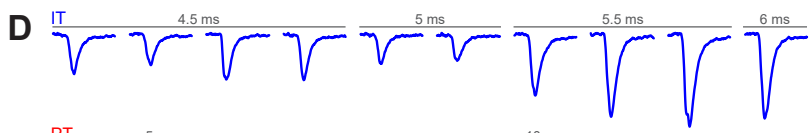

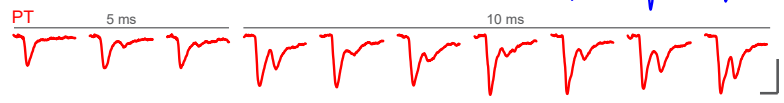

$\mathbf{F}$

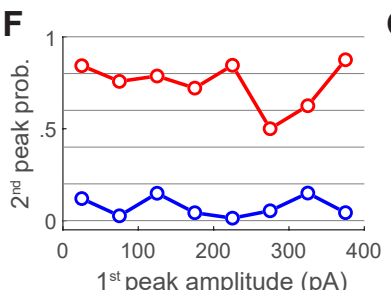

G

H
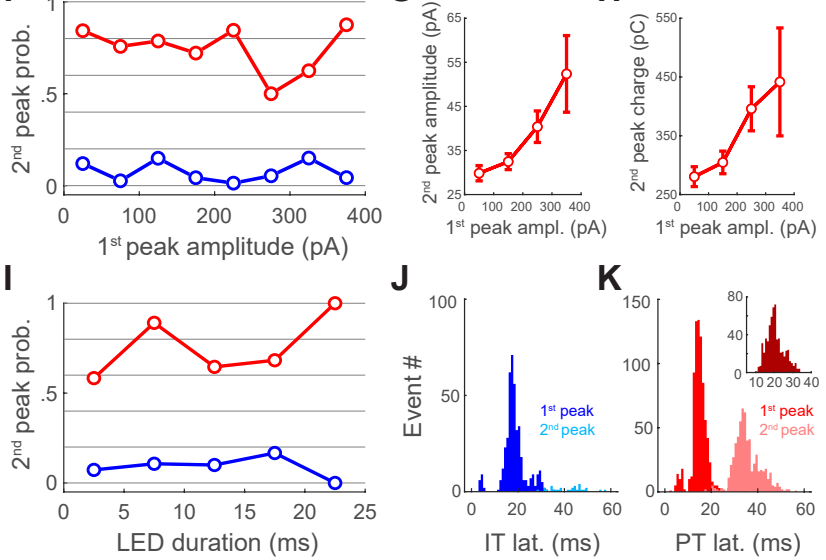

K

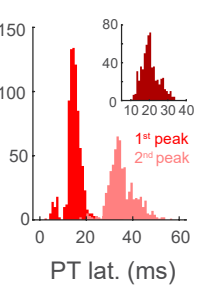

M
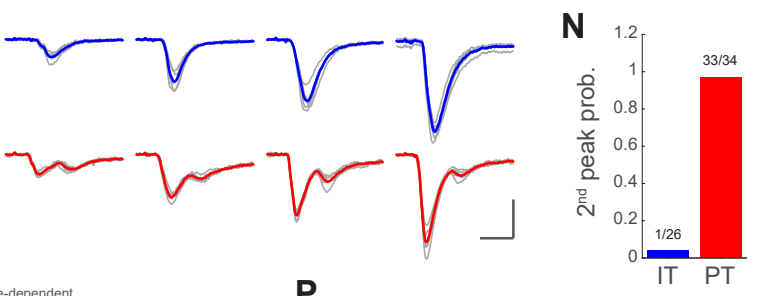

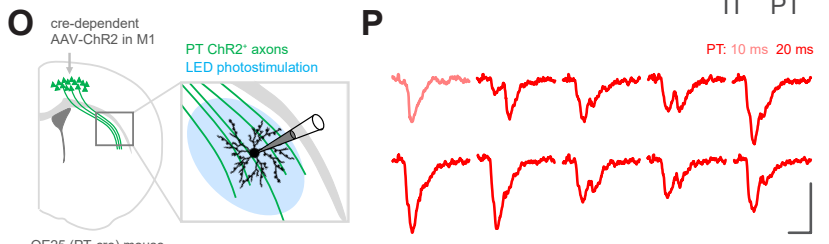

Figure 1

Morgenstern et al. 2020 

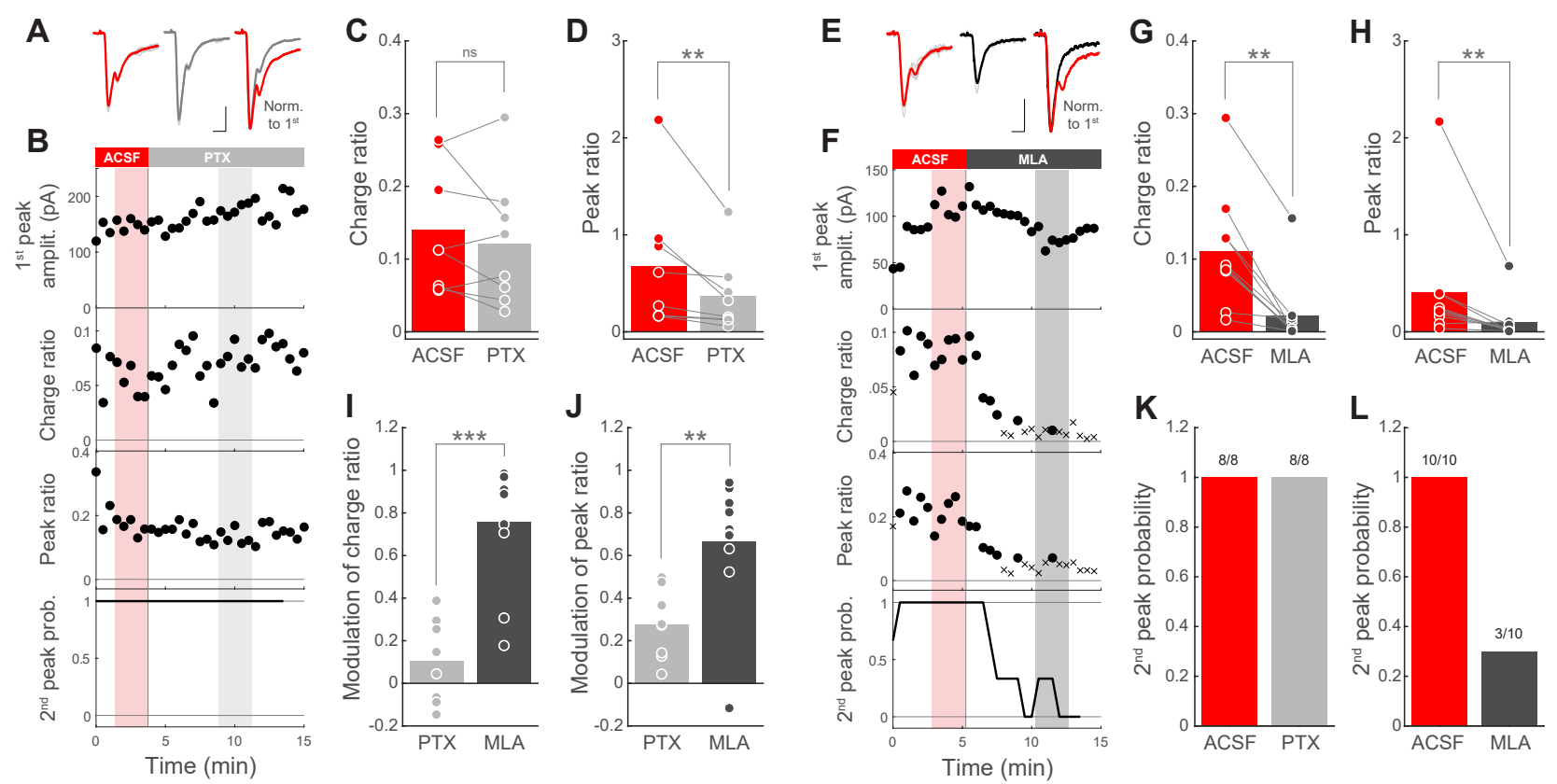

Figure 2

Morgenstern et al. 2020 

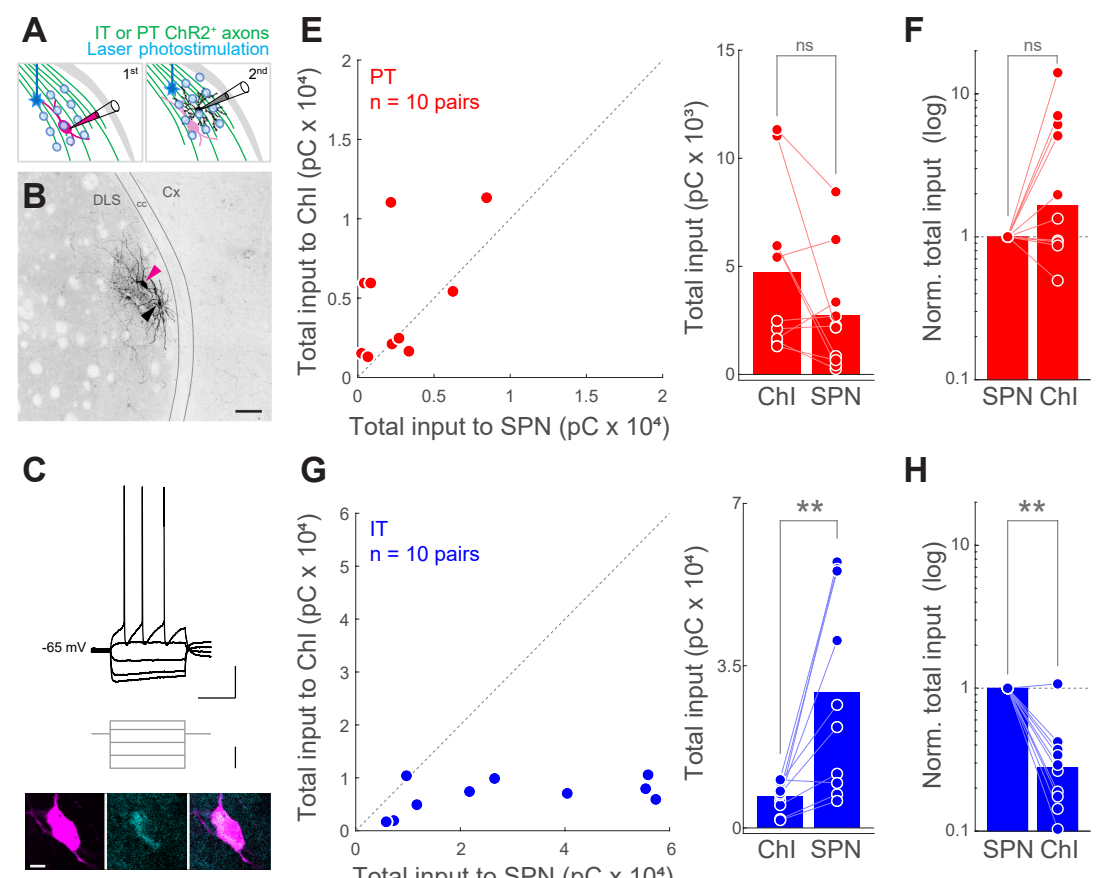

G

H
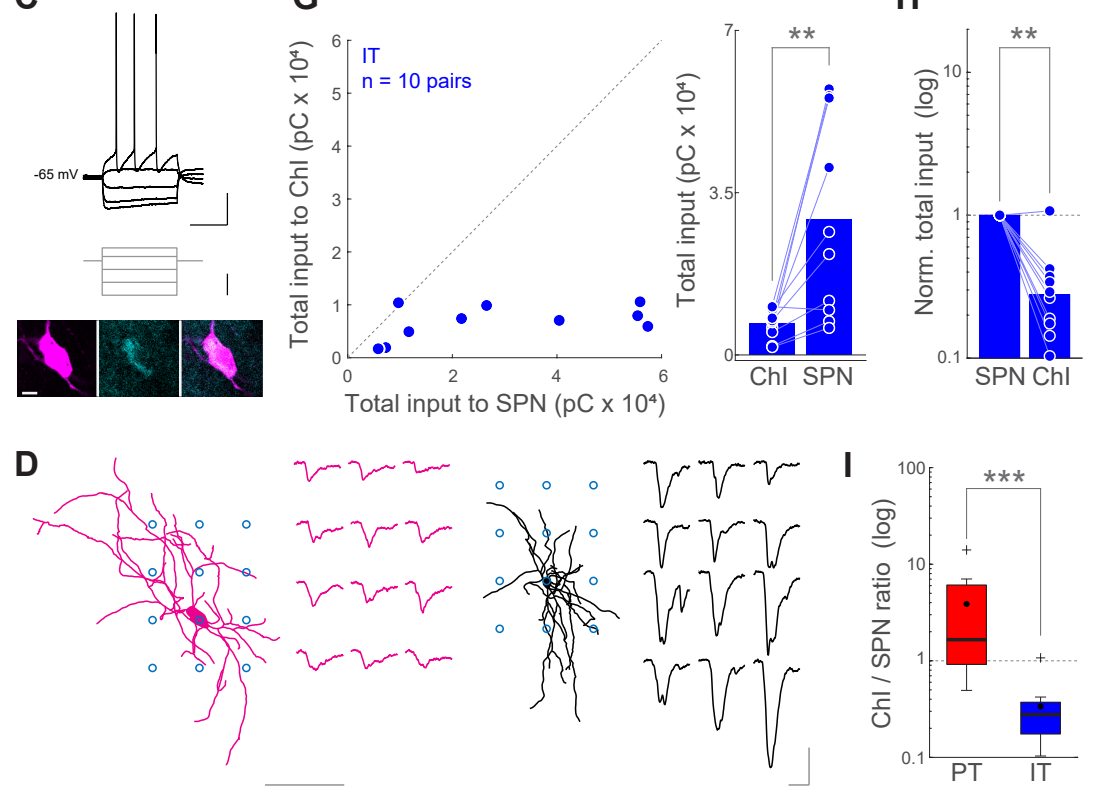

Figure 3

Morgenstern et al. 2020 

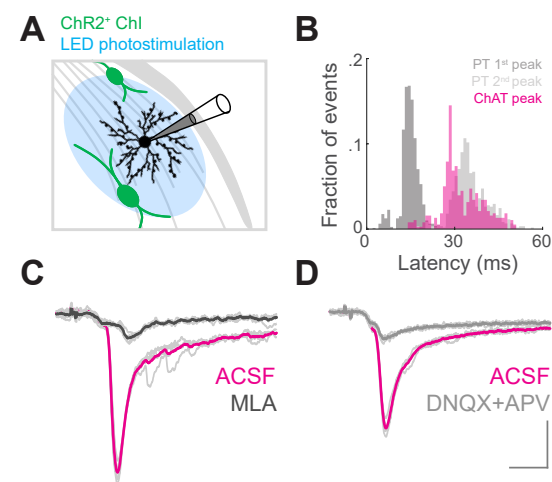

D
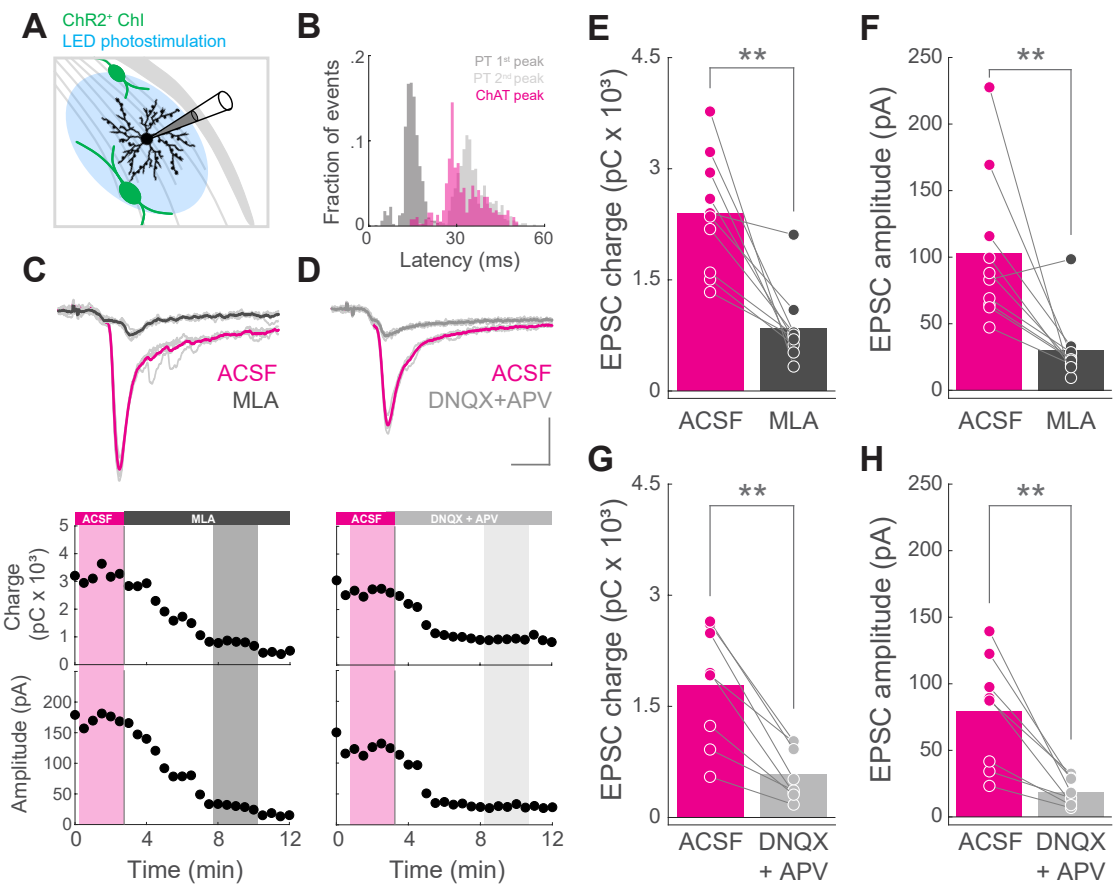

Figure 4

Morgenstern et al. 2020 

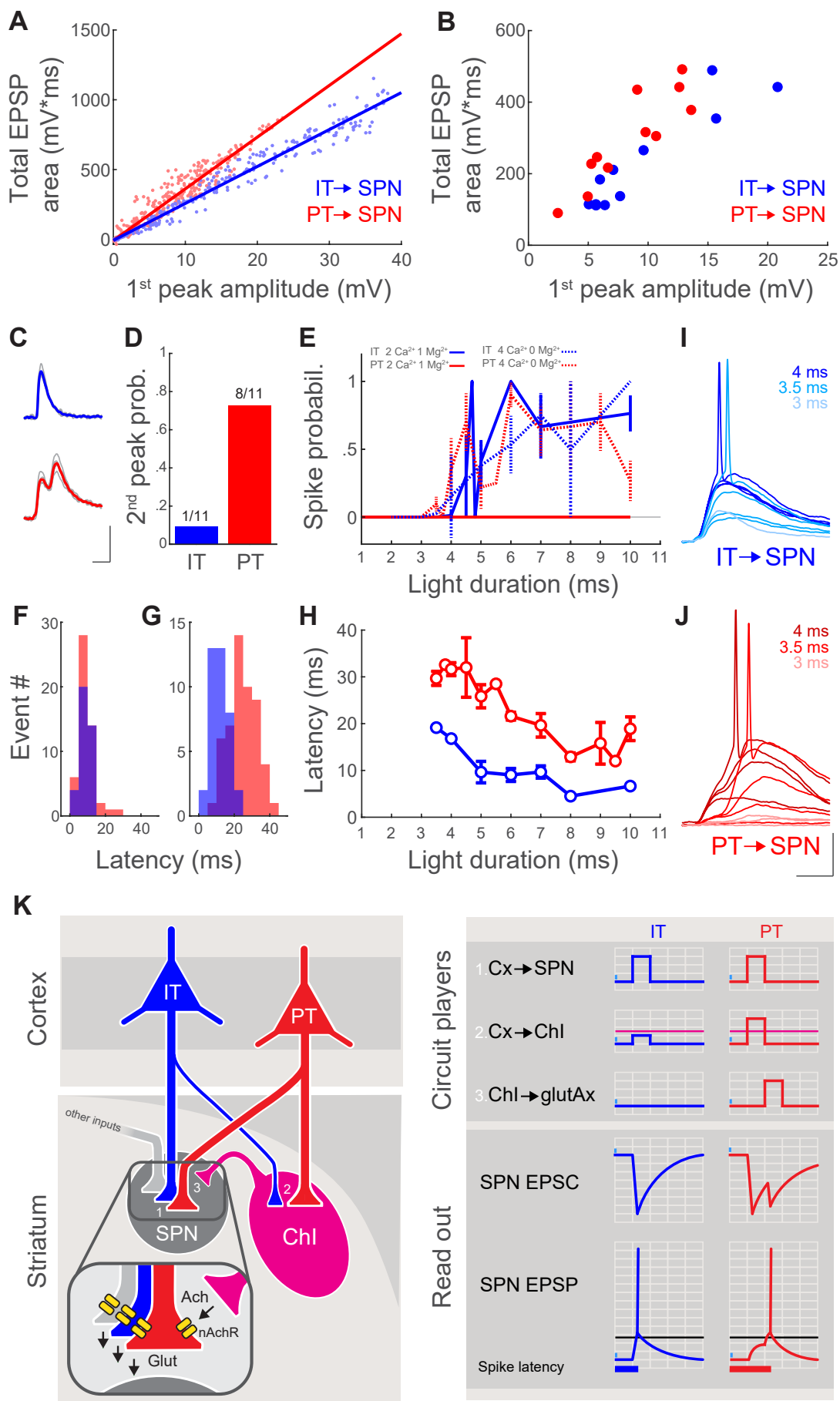

Figure 5

Morgenstern et al. 2020 